\title{
Interventions to improve discharge from acute adult mental health inpatient care to the community: systematic review and narrative synthesis
}

Natasha Tyler ${ }^{1 *}$ (D) Nicola Wright ${ }^{2}$ and Justin Waring ${ }^{1,3}$

\begin{abstract}
Background: The transition from acute mental health inpatient to community care is often a vulnerable period in the pathway, where people can experience additional risks and anxiety. Researchers globally have developed and tested a number of interventions that aim to improve continuity of care and safety in these transitions. However, there has been little attempt to compare and contrast the interventions and specify the variety of safety threats they attempt to resolve.
\end{abstract}

Methods: The study aimed to identify the evidence base for interventions to support continuity of care and safety in the transition from acute mental health inpatient to community services at the point of discharge. Electronic Databases including PsycINFO, MEDLINE, Embase, HMIC, CINAHL, IBSS, Cochrane Library Trials, ASSIA, Web of Science and Scopus, were searched between 2000 and May 2018. Peer reviewed papers were eligible for inclusion if they addressed adults admitted to an acute inpatient mental health ward and reported on health interventions relating to discharge from the acute ward to the community. The results were analysed using a narrative synthesis technique.

Results: The total number of papers from which data were extracted was 45 . The review found various interventions implemented across continents, addressing problems related to different aspects of discharge. Some interventions followed a distinct named approach (i.e. Critical Time Intervention, Transitional Discharge Model), others were grouped based on key components (i.e. peer support, pharmacist involvement). The primary problems interventions looked to address were reducing readmission, improving wellbeing, reducing homelessness, improving treatment adherence, accelerating discharge, reducing suicide. The 69 outcomes reported across studies were heterogeneous, meaning it was difficult to conduct comparative quantitative meta-analysis or synthesis.

Conclusions: The interventions reviewed are spread across a spectrum ranging from addressing a single problem within a single agency with a single solution, to multiple solutions addressing multi-agency problems. We recommend that future research attempts to improve homogeneity in outcome reporting.

Keywords: Systematic review, Care transitions, Mental health, Interventions, Discharge, Acute services, Psychiatric discharge, Hospital discharge

\footnotetext{
* Correspondence: Natasha.tyler@manchester.ac.uk

${ }^{1}$ NIHR Greater Manchester Patient Safety Translational Research Centre, The

University of Manchester, Manchester, UK

Full list of author information is available at the end of the article
}

(c) The Author(s). 2019 Open Access This article is distributed under the terms of the Creative Commons Attribution 4.0 International License (http://creativecommons.org/licenses/by/4.0/), which permits unrestricted use, distribution, and reproduction in any medium, provided you give appropriate credit to the original author(s) and the source, provide a link to the Creative Commons license, and indicate if changes were made. The Creative Commons Public Domain Dedication waiver (http://creativecommons.org/publicdomain/zero/1.0/) applies to the data made available in this article, unless otherwise stated. 


\section{Background}

The transition from acute mental health inpatient to community care is often a vulnerable period in the pathway, where people can experience additional risks to their mental health and psychological wellbeing. Previous research with service users has found discharge to be a chaotic, stressful and emotionally charged time [1]. The term "revolving door" is widely used to describe how mental health service users can repeatedly transition between hospital and community care, and then back into hospital within a very short timeframe. However, the terminology of "revolving door", within this context, has been criticised by service users and the survivor movement for situating the problem of repeated transitions with the individual rather than with the systems around them [2]. This 'circuit of care' stems not only from the person's underlying health conditions, but often from the challenges of ensuring the continuity of care following inpatient discharge. A pilot qualitative study conducted in the United Kingdom (UK) [1, 3] identified examples of these challenges including; (1) problems with medication management and maintaining concordance; (2) increased risk to self (i.e. suicide) and others (particularly family members); (3) poor information sharing between services leading to both gaps and duplication in provision; and (4) poorer mental health due to the distress caused by multiple often difficult transitions. In recent years there has been considerable research evidencing the relationship between discharge from acute mental health inpatient units and suicide [4]. Research shows, for example, that between 2005 and $201517 \%$ of people who completed suicide had recently been discharged from acute hospital services [4]. The significance of suicide as a marker of quality during and after acute care is further indicated by its routine use in many studies and evaluations of interventions to support hospital discharge, alongside other measures such as readmission and length of stay [5-8]. However, the variety of challenges that are present at this sensitive time in the service user journey transverse far beyond what can be measured solely using readmission or death by suicide rates.

Researchers globally have developed and tested a number of interventions that aim to improve the care transition. Some interventions are targeted to a particular group, i.e. to reduce the risk of post-discharge homelessness $[9,10]$. Other interventions focus on a particular source of risk to health following discharge, such as medicines management $[11,12]$. Whilst others are concerned with coordinating care, more broadly, between different agencies [13, 14].

How such interventions are configured, in terms of their cause-and-effect mechanisms, and implemented in different contexts provides additional insight about how service leaders and researchers understand and seek to address the problems of care transitions from acute mental health settings. That is, whether the source of risk is located within the individual who needs additional education or support, with the care system in terms of the problems of coordinating care, or with wider social and community factors. As such interventions are so varied, it shows that different groups articulate the challenges associated with discharge differently. It is increasingly recognised that the evaluation of quality improvement interventions, such as those for hospital discharge, should more explicitly articulate and appraise the underpinning theory of change for a given intervention (the rationale and assumptions about mechanisms that links processes and inputs to outcomes, also specifying the conditions necessary for effectiveness) [15-17].

There has been little attempt to compare and contrast the interventions and specify the variety of problems they implicitly or explicitly attempt to resolve. Previous systematic reviews of discharge interventions have been restrictive. For example, one systematic review focused only on transitional interventions that aimed to reduce readmission [18]. Another review was restricted to interventions that were delivered pre-discharge [19]. The problems each intervention hopes to address are often varied or implicit, as is the study design and outcome measures used. There has been little attempt to descriptively compare the types of interventions that exist and the quality and safety challenges that they aim to address. By removing the search restrictions we hope to compare and contrast the interventions that have been tested and look for commonalities and differences in effectiveness and in the way different researchers articulate the problems associated with discharge.

\section{Aim}

To identify and synthesise the evidence base for interventions to support continuity of care and safety in the transition from adult acute mental health inpatient service to community care services at the point of discharge.

\section{Methods \\ Study design}

Systematic review. The review follows Preferred Reporting Items for Systematic Reviews and Meta-Analyses (PRISMA) reporting guidelines. For the PRISMA checklist, see Additional file 1 . The study protocol was prospectively registered with PROSPERO (CRD42018097475).

\section{Data sources}

Medical and social science databases (including PsycINFO, MEDLINE, Embase, HMIC, CINAHL, IBSS, Cochrane Library Trials, ASSIA, Web of Science and Scopus) were searched from 1st January 2000 to May 2018. A combination of controlled vocabulary index and free text terms were used to search electronic databases, including 
terms relating to discharge (e.g. "discharge", "hospital discharge", "psychiatric hospital discharge", "transfer"), mental health (e.g. "exp mental health", "mental" disorder", "mental illness"*", "schizophr"*", "suicid"), and interventions (e.g. "exp intervention", "discharge intervention", "discharge planning) see Additional file 2 for a full list of search terms used. Where a controlled vocabulary index did not exist for a database or website, only free text terms were used. Forward and backward searches were conducted on included papers using Google Scholar. All identified references were imported to Mendeley.

\section{Selection criteria Inclusion criteria}

Papers were eligible for inclusion if they 1) addressed adults (18-65); 2) admitted to an acute inpatient mental health setting; 3 ) functional conditions (mental disorders other than dementia, and includes severe mental illness such as schizophrenia) 4) reported on health interventions or service provision; 5) interventions that aimed to improve discharge from the acute ward to the community. Papers were eligible for inclusion in the review if they were peer-reviewed, empirical studies (quantitative or qualitative design) and reported original data. We included interventions that aimed to improve the transition from in-patient to community care for an adult population. Components of the intervention could be delivered prior to discharge, shortly after discharge or could span both. Papers were not excluded based on country of origin.

\section{Exclusion criteria}

We excluded interventions not related to functional adult mental health (i.e. medical, surgical, paediatric/ older adults and organic conditions) and that were involuntary in nature (e.g. involuntary treatment orders or forensic interventions). This is because we were interested in clinical interventions that promoted safety at discharge rather than the use of legislation to forcibly transition someone from one setting to another. We excluded interventions focused on treating specific psychiatric disorders (for example, medication or specific psychotherapies) unless there was a component that specifically focused on improving the transition from in-patient to community care. Theses, editorials and opinion pieces were excluded. Studies that did not include primary data were removed (e.g. systematic reviews). If a research team reported the same study in multiple papers, only the original paper was included. The search was restricted to English language only papers.

\section{Screening}

All papers identified by the database searches were downloaded to Mendeley to remove duplicates and screened at the title/abstract level for inclusion in full-text review by one reviewer (NT). Two reviewers (NW and JW) each independently screened $20 \%$ of the titles and abstracts. Group discussions resolved any differences between the reviewers and confirmed the final included studies list.. Details of the excluded papers and reasons for exclusion are available on request.

\section{Data extraction and quality appraisal}

Data were extracted from each study into a standardised table. Data extraction was conducted by one researcher NT. As a check, one reviewer (NW) checked the extracted data from a random 10 papers. Data were extracted related to: a) Aim of study b) Disciplinary perspective c) Theoretical background d) Geographical context e) Context for study f) Method g) Sample h) Analytical approach i) Outcomes measured j) Intervention details k) Evidence of outcome/Effect of intervention l) Evidence about genesis.

The methodological quality of studies was independently assessed by one reviewer (NT) using the Mixed Methods Appraisal Tool (MMAT) [20]. Complete listings of all studies and quality appraisal scores are presented in Additional file 3.

\section{Analysis}

Due to the heterogeneity in the data and outcomes reported, statistical pooling of the data was not used. Therefore, a narrative approach to synthesising the included studies was taken. Narrative synthesis is an approach to systematic review and synthesis of findings from multiple studies that depends primarily on using words and text to explain and summarise the findings [21]. The narrative synthesis was conducted using guidance, by one reviewer (NT) [22]. A four stage process was followed; 1) developing a theory of how the intervention works, why and for whom; 2) developing a preliminary synthesis; 3) exploring the relationships between and within the studies; and 4) assessing the robustness of the synthesis. Additional file 4 illustrates the synthesis process and the tools used. Extracted data were analysed by one reviewer (NT) using narrative synthesis.

\section{Results}

\section{Data sources}

The search of the electronic databases generated 3595 hits including duplicates. Citation mapping revealed a further 36 papers which were included. One thousand six hundred sixty-two unique papers were identified; 1542 papers were excluded after screening and 120 full papers were reviewed. We excluded 75 full texts for the following reasons: (1) not related to mental health, (2) not related to an adult populations, (3) not describing an intervention (4) not including primary empirical results, (5) not an acute inpatient population (i.e. forensic or 
organic) (6) not focused on discharge. Therefore, the total number of papers from which data were extracted was 45 (Fig. 1). Table 1 presents the 45 studies included in the review, alphabetised by author name, location, intervention, population, method, findings and the problems they aim to address. We first of all grouped the studies in terms of design and populations. We then grouped the studies by the type of interventions, then the problems they aim to address. Finally, we collate the outcomes reported and the facilitators and challenges reported.

\section{Study design and population}

The majority of the studies included in the review were conducted in high income countries, primarily the United States of America (USA), UK, Canada and Australia. However, a limited number were conducted in low and middle-income countries, such as Iran and China (see Fig. 2).

The methodological design used in the papers varied considerably, the most common design used was a randomised controlled trial; which is often considered the highest quality research [23]. However, many of the papers were evaluation studies, often a mixed methods review of the effectiveness of a pilot or controlled studies using a non-randomised comparison group, see Fig. 3.

Given the variation in design, there was also variation in the number of participants recruited in each study. Whilst most studies contained more than 20 participants (93\%), the majority contained less than 200 (78\%). Only $7 \%$ of studies had more than 400 participants. It must be noted that the participant numbers often included the control group; in which case only half of the participant group received the intervention.

\section{Types of interventions}

During the preliminary synthesis we used the clustering method to identify similarities between interventions (Table 2). Table 2 highlights this clustering, for some interventions this was explicit due to the use of a name for a distinct approach (i.e. Critical Time Intervention, Transitional Discharge Model) for others this involved implicitly grouping interventions based on key components (i.e. peer support, pharmacist involvement).

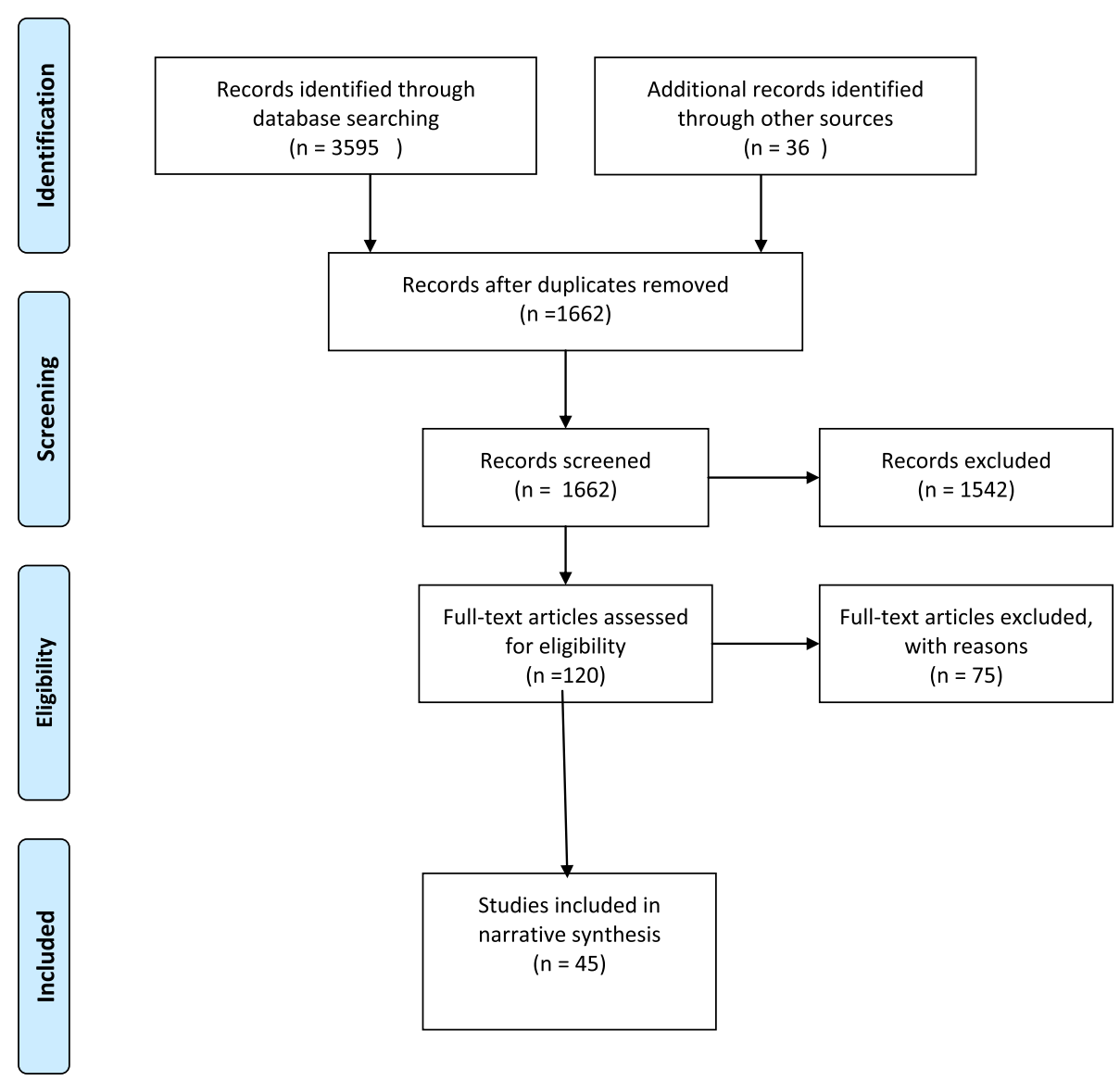

Fig. 1 PRISMA flow chart. PRISMA flow chart to report numbers of included and excluded papers at each stage 
Table 1 Tabulation and description of the studies used in the systematic review

\begin{tabular}{|c|c|c|c|c|c|c|c|}
\hline ID & $\begin{array}{l}\text { Authors and } \\
\text { year }\end{array}$ & $\begin{array}{l}\text { Location and } \\
\text { setting }\end{array}$ & Intervention & Participants & Method & Main findings & $\begin{array}{l}\text { Main aim/ problem } \\
\text { to address }\end{array}$ \\
\hline 1 & $\begin{array}{l}\text { Abraham et al. } \\
\text { (2017) }\end{array}$ & $\begin{array}{l}\text { USA, } 1 \text { urban } \\
\text { psychiatric hospital }\end{array}$ & $\begin{array}{l}\text { Pharmacist } \\
\text { involvement to } \\
\text { improve care } \\
\text { co-ordination }\end{array}$ & $\begin{array}{l}16 \text { health } \\
\text { professionals, } 6 \\
\text { patients, } 20 \\
\text { patient charts } \\
\text { (SMl patients) }\end{array}$ & $\begin{array}{l}\text { Evaluation- } \\
\text { interviews and } \\
\text { observations of } \\
\text { charts }\end{array}$ & $\begin{array}{l}\text { Increased pharmacist } \\
\text { involvement in LAl } \\
\text { care coordination may } \\
\text { contribute to bridging } \\
\text { gaps in medication } \\
\text { adherence to optimize } \\
\text { treatment outcomes. }\end{array}$ & $\begin{array}{l}\text { To support long-term } \\
\text { medication adherence } \\
\text { and patient outcomes }\end{array}$ \\
\hline 2 & $\begin{array}{l}\text { Attfield et al. } \\
\text { (2017) }\end{array}$ & UK, 2 trusts & $\begin{array}{l}\text { Diagnostic-driven } \\
\text { Integrated Care } \\
\text { Pathways (ICPS) }\end{array}$ & $\begin{array}{l}\text { A random } \\
\text { sample of } 400 \\
\text { service users }\end{array}$ & $\begin{array}{l}\text { Retrospective } \\
\text { case comparison } \\
\text { study }\end{array}$ & $\begin{array}{l}\text { The ICP Trust had a } \\
13.5 \text { day shorter } \\
\text { average length of stay, } \\
\text { (statistically } \\
\text { significant). No } \\
\text { significant differences } \\
\text { in readmission or } \\
\text { 7-day follow-up. }\end{array}$ & $\begin{array}{l}\text { Reducing unnecessary } \\
\text { tests, interventions } \\
\text { and duplication } \\
\text { within the care } \\
\text { process }\end{array}$ \\
\hline 3 & $\begin{array}{l}\text { Bauer et al. } \\
\text { (2012) }\end{array}$ & $\begin{array}{l}\text { Germany, } 1 \\
\text { hospital }\end{array}$ & $\begin{array}{l}\text { SMS-based } \\
\text { maintenance } \\
\text { intervention }\end{array}$ & $\begin{array}{l}165 \text { females. } \\
\text { Eating } \\
\text { disorders }\end{array}$ & $\mathrm{RCT}$ & $\begin{array}{l}\text { Somewhat significant } \\
\text { difference in } \\
\text { readmission } \\
\text { (depending on } \\
\text { analysis). Significant } \\
\text { difference in } \\
\text { treatment utilisation. }\end{array}$ & Maintain treatment \\
\hline 4 & $\begin{array}{l}\text { Bennewith } \\
\text { et al. (2014) }\end{array}$ & $\begin{array}{l}\text { UK, } 3 \text { inpatient } \\
\text { wards in southwest } \\
\text { England, mixed } \\
\text { urban/rural }\end{array}$ & $\begin{array}{l}\text { A contact-based inter- } \\
\text { vention for people } \\
\text { recently discharged } \\
\text { (letters sent to sus) }\end{array}$ & $\begin{array}{l}102 \text { patients } \\
\text { received a } \\
\text { letter, } 45 \\
\text { received all } \\
\text { letters }\end{array}$ & $\begin{array}{l}\text { Pilot case study. } \\
\text { Interviews, } \\
\text { analysis of } \\
\text { outcomes } \\
\text { (readmission) }\end{array}$ & $\begin{array}{l}\text { Post-discharge, } \\
\text { qualitative interviews } \\
\text { with service users } \\
\text { showed that most } \\
\text { already felt adequately } \\
\text { supported and the } \\
\text { intervention added } \\
\text { little to this. }\end{array}$ & $\begin{array}{l}\text { To reduce suicide } \\
\text { post-discharge by } \\
\text { providing social } \\
\text { connectedness }\end{array}$ \\
\hline 5 & $\begin{array}{l}\text { Bonsack et al. } \\
\text { (2016) }\end{array}$ & $\begin{array}{l}\text { Switzerland, } 1 \\
\text { psychiatric hospital }\end{array}$ & $\begin{array}{l}\text { Transitional case } \\
\text { management }\end{array}$ & $\begin{array}{l}51 \\
\text { intervention, } \\
51 \text { control }\end{array}$ & $\mathrm{RCT}$ & $\begin{array}{l}\text { Increased short-term } \\
\text { rate of engagement } \\
\text { with ambulatory care } \\
\text { despite no differences } \\
\text { between the two } \\
\text { groups after } 3 \text { months } \\
\text { of follow-up. Interven- } \\
\text { tion did not signifi- } \\
\text { cantly reduce the rate } \\
\text { of readmissions during } \\
\text { the first year following } \\
\text { discharge. }\end{array}$ & $\begin{array}{l}\text { Improve engagement } \\
\text { with care, reduce } \\
\text { readmission }\end{array}$ \\
\hline 6 & $\begin{array}{l}\text { Botha et al. } \\
(2018)\end{array}$ & $\begin{array}{l}\text { South Africa, } 1 \\
\text { hospital }\end{array}$ & $\begin{array}{l}\text { 90-day transitional } \\
\text { care intervention (four } \\
\text { phone calls and one } \\
\text { home visit, focusing } \\
\text { on maintaining } \\
\text { adherence, } \\
\text { appointment } \\
\text { reminders and } \\
\text { psychoeducation.) }\end{array}$ & $\begin{array}{l}60 \text { male } \\
\text { patients }\end{array}$ & $\begin{array}{l}\text { Retrospective } \\
\text { comparison to } \\
\text { matched control } \\
\text { group }\end{array}$ & $\begin{array}{l}\text { No effect on } \\
\text { readmission rates in } \\
\text { this setting. }\end{array}$ & $\begin{array}{l}\text { Bridge gap, reduce } \\
\text { readmissions }\end{array}$ \\
\hline 7 & Chen (2014) & $\begin{array}{l}\text { USA, all of the } \\
\text { community } \\
\text { agencies providing } \\
\text { CTI in NYC (4) }\end{array}$ & $\begin{array}{l}\text { Community support } \\
\text { in critical time } \\
\text { intervention (CTI)a } \\
\text { time-limited, short- } \\
\text { term psychosocial } \\
\text { rehabilitation. } \\
\text { Program designed to } \\
\text { facilitate the critical } \\
\text { transition from } \\
\text { institutional to } \\
\text { community settings }\end{array}$ & $12 \mathrm{CTI}$ workers & Interviews & $\begin{array}{l}\text { CTI workers self- } \\
\text { identified as "extra } \\
\text { support" to develop } \\
\text { community ties that } \\
\text { will help clients sus- } \\
\text { tain stable housing. } \\
\text { Propose a transient } \\
\text { triangular relationship } \\
\text { model, involving three } \\
\text { dyadic relationships } \\
\text { (worker-client, worker- } \\
\text { primary support, pri- } \\
\text { mary support client). }\end{array}$ & $\begin{array}{l}\text { To facilitate effective } \\
\text { transitional services } \\
\text { and enhance } \\
\text { continuity of care. } \\
\text { Breaking the vicious } \\
\text { cycle between } \\
\text { institutionalization } \\
\text { and homelessness }\end{array}$ \\
\hline
\end{tabular}


Table 1 Tabulation and description of the studies used in the systematic review (Continued)

\begin{tabular}{|c|c|c|c|c|c|c|c|}
\hline ID & $\begin{array}{l}\text { Authors and } \\
\text { year }\end{array}$ & $\begin{array}{l}\text { Location and } \\
\text { setting }\end{array}$ & Intervention & Participants & Method & Main findings & $\begin{array}{l}\text { Main aim/ problem } \\
\text { to address }\end{array}$ \\
\hline 8 & D'Souza (2002) & $\begin{array}{l}\text { Australia, rural } \\
\text { hospital }\end{array}$ & $\begin{array}{l}\text { Telemedicine (psycho- } \\
\text { educational } \\
\text { programme and MDT } \\
\text { videoconferencing } \\
\text { post-discharge) }\end{array}$ & $\begin{array}{l}51 \text { ( } 24 \\
\text { intervention, } \\
27 \text { control) } \\
\text { male and } \\
\text { female }\end{array}$ & Controlled study & $\begin{array}{l}\text { More side effects in } \\
\text { control group, more } \\
\text { treatment adherence } \\
\text { and satisfaction in } \\
\text { intervention group. }\end{array}$ & $\begin{array}{l}\text { Improve treatment } \\
\text { adherence }\end{array}$ \\
\hline 9 & $\begin{array}{l}\text { De Leo and } \\
\text { Heller (2007) }\end{array}$ & $\begin{array}{l}\text { Australia, } 1 \\
\text { psychiatric } \\
\text { inpatient unit }\end{array}$ & $\begin{array}{l}\text { Intensive case } \\
\text { management follow } \\
\text { up of high risk people } \\
\text { (ICM was weekly face- } \\
\text { to-face contact with } \\
\text { community case man- } \\
\text { ager and telephone } \\
\text { calls from counsellors) }\end{array}$ & $\begin{array}{l}60 \text { male service } \\
\text { users with a } \\
\text { history of } \\
\text { suicide } \\
\text { attempts }\end{array}$ & $\begin{array}{l}\text { RCT (TAU or } \\
\text { intervention) }\end{array}$ & $\begin{array}{l}\text { People in ICM had } \\
\text { lower depression } \\
\text { scores, suicidal } \\
\text { ideation, QoL, more } \\
\text { contact with services, } \\
\text { better relationships } \\
\text { with therapists and } \\
\text { were satisfied with } \\
\text { service. }\end{array}$ & $\begin{array}{l}\text { A solution to the } \\
\text { reduced care } \\
\text { following discharge } \\
\text { that is linked to } \\
\text { suicide. }\end{array}$ \\
\hline 10 & $\begin{array}{l}\text { Exbrayat et al. } \\
(2017)\end{array}$ & $\begin{array}{l}\text { France, single } \\
\text { centre }\end{array}$ & $\begin{array}{l}\text { Telephone } \\
\text { follow-up } 8,30 \text { and } 60 \\
\text { days post attempted } \\
\text { suicide }\end{array}$ & $\begin{array}{l}436 \text { patients } \\
\text { (387 control } \\
\text { patients who } \\
\text { were matched } \\
\text { from pre- } \\
\text { intervention } \\
\text { records) }\end{array}$ & Controlled study & $\begin{array}{l}\text { Very early telephone } \\
\text { follow-up of our pa- } \\
\text { tients effectively re- } \\
\text { duced recidivism and } \\
\text { seemed to be the only } \\
\text { protective factor } \\
\text { against repeated sui- } \\
\text { cide attempt. }\end{array}$ & $\begin{array}{l}\text { To reduce suicide } \\
\text { attempts post- } \\
\text { discharge }\end{array}$ \\
\hline 11 & $\begin{array}{l}\text { Forchuk et al. } \\
\text { (2005) }\end{array}$ & $\begin{array}{l}\text { Canada, } 26 \text { wards, } \\
4 \text { hospitals }\end{array}$ & $\begin{array}{l}\text { Transitional discharge } \\
\text { model (TDM) }\end{array}$ & 390 & $\begin{array}{l}\text { Randomised } \\
\text { clinical trial } \\
\text { using a cluster } \\
\text { design }\end{array}$ & $\begin{array}{l}\text { Costs and quality of } \\
\text { life were not } \\
\text { significantly improved } \\
\text { post-discharge com- } \\
\text { pared with the control } \\
\text { group. Although not } \\
\text { predicted a priori, } \\
\text { intervention subjects } \\
\text { were discharged an } \\
\text { average of } 116 \text { days } \\
\text { earlier per person. }\end{array}$ & $\begin{array}{l}\text { Reduce bed } \\
\text { occupancy, improve } \\
\text { quality of life }\end{array}$ \\
\hline 12 & $\begin{array}{l}\text { Forchuk et al. } \\
\text { (2008) }\end{array}$ & Canada, 1 hospital & $\begin{array}{l}\text { Intervention to } \\
\text { prevent } \\
\text { homelessness- } \\
\text { immediate assistance } \\
\text { in accessing housing } \\
\text { and assistance in } \\
\text { paying their first and } \\
\text { last month's rent }\end{array}$ & $\begin{array}{l}14 \text { participants } \\
\text { at risk of being } \\
\text { discharged } \\
\text { without } \\
\text { housing ( } 7 \text { in } \\
\text { intervention } \\
\text { group) }\end{array}$ & $\begin{array}{l}\mathrm{RCT} \text {, incl. } \\
\text { interviews }\end{array}$ & $\begin{array}{l}\text { All intervention group } \\
\text { maintained housing } \\
\text { after } 3 \text { and } 6 \text { months. } \\
\text { All but one individual } \\
\text { in the control group } \\
\text { remained homeless } \\
\text { after } 3 \text { and } 6 \text { months. } \\
\text { Results of this pilot } \\
\text { were so dramatic that } \\
\text { randomizing to the } \\
\text { control group was } \\
\text { discontinued }\end{array}$ & $\begin{array}{l}\text { To reduce discharge } \\
\text { from inpatient wards } \\
\text { to shelters or the } \\
\text { street }\end{array}$ \\
\hline 13 & $\begin{array}{l}\text { Forchuk et al. } \\
\text { (2012) }\end{array}$ & Canada, 6 hospitals & $\begin{array}{l}\text { Transitional } \\
\text { relationship model } \\
\text { (TRM) (providing } \\
\text { hospital staff } \\
\text { involvement until a } \\
\text { therapeutic } \\
\text { relationship has been } \\
\text { established with a } \\
\text { community care } \\
\text { provider as well as } \\
\text { peer support.) }\end{array}$ & $\begin{array}{l}\text { No participant } \\
\text { numbers as } \\
\text { ethnographic } \\
\text { analysis. } 14 \text { A } \\
\text { wards, } 12 \text { B } \\
\text { wards and } 10 \\
\text { C wards. }\end{array}$ & $\begin{array}{l}\text { A quasi- } \\
\text { experimental, } \\
\text { action-oriented } \\
\text { research design }\end{array}$ & $\begin{array}{l}\text { Staged large-scale im- } \\
\text { plementation allowed } \\
\text { for iterative improve- } \\
\text { ments to the } \\
\text { model leading to } \\
\text { positive outcomes. } \\
\text { This study highlights } \\
\text { the need to address } \\
\text { work environment } \\
\text { issues, particularly } \\
\text { inter-professional } \\
\text { teams. }\end{array}$ & $\begin{array}{l}\text { To improve staff } \\
\text { uptake of } \\
\text { interventions }\end{array}$ \\
\hline 14 & $\begin{array}{l}\text { Forchuk et al. } \\
\text { (2013) }\end{array}$ & $\begin{array}{l}\text { Canada, all patients } \\
\text { in Ontario at risk of } \\
\text { homelessness, } 1 \\
\text { acute care hospital, } \\
1 \text { territory }\end{array}$ & $\begin{array}{l}\text { Intervention to } \\
\text { prevent homelessness } \\
- \\
\text { Pre-discharge } \\
\text { assistance in securing } \\
\text { housing }\end{array}$ & $\begin{array}{l}112 \text { men and } \\
107 \text { women at } \\
\text { risk of } \\
\text { homelessness } \\
\text { post-discharge }\end{array}$ & $\begin{array}{l}\text { Programme } \\
\text { evaluation } \\
\text { design- } \\
\text { interviews, focus } \\
\text { groups }\end{array}$ & $\begin{array}{l}\text { The results highlight } \\
\text { several benefits of the } \\
\text { intervention and show } \\
\text { that homelessness can } \\
\text { be reduced by } \\
\text { connecting housing } \\
\text { support, income }\end{array}$ & $\begin{array}{l}\text { To stop people being } \\
\text { discharged to street } \\
\text { or shelters }\end{array}$ \\
\hline
\end{tabular}


Table 1 Tabulation and description of the studies used in the systematic review (Continued)

\begin{tabular}{|c|c|c|c|c|c|c|c|}
\hline $\mathrm{ID}$ & $\begin{array}{l}\text { Authors and } \\
\text { year }\end{array}$ & $\begin{array}{l}\text { Location and } \\
\text { setting }\end{array}$ & Intervention & Participants & Method & Main findings & $\begin{array}{l}\text { Main aim/ problem } \\
\text { to address }\end{array}$ \\
\hline & & & & & & $\begin{array}{l}\text { support, and } \\
\text { psychiatric care. }\end{array}$ & \\
\hline 15 & $\begin{array}{l}\text { Ghadiri Vasfi } \\
\text { et al. (2015) }\end{array}$ & Iran, 1 hospital & $\begin{array}{l}\text { Aftercare Services } \\
\text { (three components: } \\
\text { follow-up } \\
\text { Care (home visits or } \\
\text { telephone follow-up), } \\
\text { family psychoeduca- } \\
\text { tion, And social skills } \\
\text { training for patients.) }\end{array}$ & $\begin{array}{l}120 \text { patients } \\
\text { (schizophrenia } \\
\text { and bipolar) } \\
\text { ages } 15-65.60 \\
\text { control }\end{array}$ & RCT & $\begin{array}{l}\text { The cumulative } \\
\text { number of } \\
\text { hospitalizations during } \\
\text { the follow-up period } \\
\text { was } 55 \text { for the control } \\
\text { group and } 26 \text { for the } \\
\text { intervention group. } \\
\text { Length of stay was } \\
\text { significantly greater in } \\
\text { the control group. Psy- } \\
\text { chopathology was sig- } \\
\text { nificantly less severe in } \\
\text { intervention group } \\
\text { compared with the } \\
\text { control }\end{array}$ & $\begin{array}{l}\text { Reducing } \\
\text { readmissions and } \\
\text { length of stay }\end{array}$ \\
\hline 16 & $\begin{array}{l}\text { Hampson } \\
\text { et al. (2000) }\end{array}$ & $\begin{array}{l}\text { UK, } 1 \text { trust (North } \\
\text { Nottingham and } \\
\text { Hucknall) }\end{array}$ & $\begin{array}{l}\text { Community Link } \\
\text { Team (CLT) to } \\
\text { facilitate early } \\
\text { discharge- team- } \\
\text { based service offering } \\
\text { intensive support dur- } \\
\text { ing the day }\end{array}$ & $\begin{array}{l}142 \text { (all } \\
\text { admissions to } \\
\text { team in } 12 \\
\text { month period) }\end{array}$ & $\begin{array}{l}\text { Retrospective } \\
\text { comparison }\end{array}$ & $\begin{array}{l}\text { Median length of stay } \\
\text { during CLT project } \\
\text { was } 19 \text { days, a highly } \\
\text { significant reduction } \\
\text { from } 36 \text { days in the } \\
\text { NABUS study. Cannot } \\
\text { be attributed to team } \\
\text { but justifies a RCT to } \\
\text { test this hypothesis, }\end{array}$ & $\begin{array}{l}\text { To speed up } \\
\text { discharge due to } \\
\text { costs to provider and } \\
\text { patients }\end{array}$ \\
\hline 17 & $\begin{array}{l}\text { Hanrahan } \\
\text { et al. (2014) }\end{array}$ & USA, 1 hospital & $\begin{array}{l}\text { Transitional care } \\
\text { model (TCM) }\end{array}$ & 40 (20 control) & $\mathrm{RCT}$ & $\begin{array}{l}\text { The intervention } \\
\text { group showed higher } \\
\text { medical and } \\
\text { psychiatric } \\
\text { rehospitalisation than } \\
\text { the control group. } \\
\text { Emergency room use } \\
\text { lower for intervention } \\
\text { group but not } \\
\text { statistically significant. } \\
\text { Continuity of care } \\
\text { with primary care } \\
\text { appointments were } \\
\text { significantly higher for } \\
\text { the intervention } \\
\text { group. The } \\
\text { intervention group's } \\
\text { general health } \\
\text { improved but was not } \\
\text { significant }\end{array}$ & $\begin{array}{l}\text { Reduce transition } \\
\text { failures }\end{array}$ \\
\hline 18 & $\begin{array}{l}\text { Hegedus et al. } \\
\text { (2018) }\end{array}$ & $\begin{array}{l}\text { Switzerland, } 2 \\
\text { wards, } 1 \text { hospital }\end{array}$ & $\begin{array}{l}\text { Short transitional } \\
\text { intervention in } \\
\text { psychiatry (step) }\end{array}$ & $\begin{array}{l}14 \text { control, } 15 \\
\text { intervention }\end{array}$ & $\begin{array}{l}\text { Quasi- } \\
\text { experimental } \\
\text { pilot study to } \\
\text { determine the } \\
\text { feasibility of the } \\
\text { intervention, }\end{array}$ & $\begin{array}{l}\text { The intervention did } \\
\text { not affect primary or } \\
\text { secondary outcomes; } \\
\text { however, it was } \\
\text { shown to be feasible, } \\
\text { and patients' feedback } \\
\text { highlighted the } \\
\text { importance of post- } \\
\text { discharge contact } \\
\text { sessions. }\end{array}$ & $\begin{array}{l}\text { Prepare patients for } \\
\text { situation outside of } \\
\text { hospital }\end{array}$ \\
\hline 19 & $\begin{array}{l}\text { Hengartner } \\
\text { et al. (2015) }\end{array}$ & $\begin{array}{l}\text { Switzerland, } 1 \\
\text { catchment area, } \\
\text { which is an urban/ } \\
\text { suburban area of } \\
\text { high-level resources } \\
\text { near the city of } \\
\text { Zurich }\end{array}$ & $\begin{array}{l}\text { Post-discharge } \\
\text { network coordination }\end{array}$ & 3 patients & $\begin{array}{l}\text { Case studies- } \\
\text { narrative review } \\
\text { and qualitative } \\
\text { analysis of three } \\
\text { patients who } \\
\text { participated in } \\
\text { the program }\end{array}$ & $\begin{array}{l}\text { Case reports revealed } \\
\text { that patients' social } \\
\text { networks are small } \\
\text { and their } \\
\text { relationships are } \\
\text { commonly conflictual } \\
\text { and unstable. }\end{array}$ & $\begin{array}{l}\text { Reducing } \\
\text { readmission, } \\
\text { improving mental } \\
\text { health and } \\
\text { psychosocial } \\
\text { functioning. Improve } \\
\text { hospital discharge } \\
\text { planning and to ease }\end{array}$ \\
\hline
\end{tabular}


Table 1 Tabulation and description of the studies used in the systematic review (Continued)

\begin{tabular}{|c|c|c|c|c|c|c|c|}
\hline $\mathrm{ID}$ & $\begin{array}{l}\text { Authors and } \\
\text { year }\end{array}$ & $\begin{array}{l}\text { Location and } \\
\text { setting }\end{array}$ & Intervention & Participants & Method & Main findings & $\begin{array}{l}\text { Main aim/ problem } \\
\text { to address }\end{array}$ \\
\hline & & & & & & & the transition \\
\hline 20 & $\begin{array}{l}\text { Hengartner } \\
\text { et al. (2016) }\end{array}$ & $\begin{array}{l}\text { Switzerland, } 1 \\
\text { catchment area, } \\
\text { which is an urban/ } \\
\text { suburban area of } \\
\text { high-level resources } \\
\text { near the city of } \\
\text { Zurich }\end{array}$ & $\begin{array}{l}\text { Post-discharge } \\
\text { network coordination }\end{array}$ & 151 patients & $\begin{array}{l}\mathrm{RCT} \text { using } \\
\text { parallel group } \\
\text { blocking }\end{array}$ & $\begin{array}{l}\text { In the short-term, no } \\
\text { significant effect } \\
\text { emerged in any out- } \\
\text { come. In the long } \\
\text { term the two groups } \\
\text { did not differ signifi- } \\
\text { cantly with rate and } \\
\text { duration of rehospitali- } \\
\text { sation. The interven- } \\
\text { tion did not reduce } \\
\text { psychiatric symptoms, } \\
\text { did not improve social } \\
\text { support, and did not } \\
\text { improve quality of life. }\end{array}$ & $\begin{array}{l}\text { Reducing } \\
\text { readmission, } \\
\text { improving mental } \\
\text { health and } \\
\text { psychosocial } \\
\text { functioning. Improve } \\
\text { hospital discharge } \\
\text { planning and to ease } \\
\text { the transition }\end{array}$ \\
\hline 21 & $\begin{array}{l}\text { Herman et al. } \\
\text { (2011) }\end{array}$ & $\begin{array}{l}\text { USA, } 2 \text { transitional } \\
\text { residences in } \\
\text { hospital grounds } \\
\text { metropolitan area }\end{array}$ & $\begin{array}{l}\text { Critical Time } \\
\text { Intervention to } \\
\text { prevent homelessness }\end{array}$ & $\begin{array}{l}150 \text { previously } \\
\text { homeless men } \\
\text { and women } \\
\text { with SMI }\end{array}$ & $\mathrm{RCT}$ & $\begin{array}{l}\text { CTI group had less } \\
\text { homelessness than } \\
\text { TAU }\end{array}$ & $\begin{array}{l}\text { Reduce homelessness } \\
\text { following discharge }\end{array}$ \\
\hline 22 & $\begin{array}{l}\text { Jenson et al. } \\
\text { (2010) }\end{array}$ & $\begin{array}{l}\text { Canada, poor city, } \\
\text { high } \\
\text { unemployment, } 1 \\
\text { acute ward and } 1 \\
\text { community service } \\
\text { provider within } \\
\text { same region }\end{array}$ & $\begin{array}{l}\text { Community-Based } \\
\text { Discharge Planning } \\
\text { (in-reach model- } \\
\text { discharge planner } \\
\text { based in community } \\
\text { visits ward daily) }\end{array}$ & $\begin{array}{l}36 \text { service } \\
\text { users }\end{array}$ & $\begin{array}{l}\text { Single group } \\
\text { programme } \\
\text { evaluation, } \\
\text { analysis of } \\
\text { admin data and } \\
\text { interview with } \\
\text { clients }\end{array}$ & $\begin{array}{l}\text { Readmission rates } \\
\text { were } 40 \% \text { lower in the } \\
\text { year following } \\
\text { the change in service } \\
\text { delivery model. This } \\
\text { change was } \\
\text { statistically significant. }\end{array}$ & $\begin{array}{l}\text { To shift mental health } \\
\text { services from } \\
\text { institution to } \\
\text { community }\end{array}$ \\
\hline 23 & $\begin{array}{l}\text { Juven-Wetzler } \\
\text { et al. (2012) }\end{array}$ & Israel, 1 ward & $\begin{array}{l}\text { "Continuation of Care" } \\
\text { model (continuing } \\
\text { follow-up in the ward, } \\
\text { by the same staff, in- } \\
\text { stead of being re- } \\
\text { ferred to the } \\
\text { outpatient } \\
\text { department.) }\end{array}$ & $\begin{array}{l}35 \text { service } \\
\text { users }\end{array}$ & $\begin{array}{l}\text { Pre and post } \\
\text { within } \\
\text { participant } \\
\text { design }\end{array}$ & $\begin{array}{l}\text { The number of } \\
\text { hospitalizations in the } \\
18 \text { months following } \\
\text { the index } \\
\text { hospitalization was } \\
1.79 \_3.51 \text { as } \\
\text { compared to } 4.67 \text { _- } \\
1.79 \text { before the index } \\
\text { hospitalization ( } p= \\
0.0002 \text { ), and the } \\
\text { number of days of } \\
\text { hospitalization } \\
18 \text { months after was } \\
24 \text { _ } 41.65 \text { as } \\
\text { compared to } 119.71 \text {. }\end{array}$ & $\begin{array}{l}\text { To reduce length of } \\
\text { stay and readmission }\end{array}$ \\
\hline 24 & $\begin{array}{l}\text { Kariel-Lauer } \\
(2000)\end{array}$ & Israel, 1 hospital & $\begin{array}{l}\text { Re-entry group (short- } \\
\text { term group meetings- } \\
\text { psychoeducational } \\
\text { approach) }\end{array}$ & $\begin{array}{l}75 \text { participants } \\
\text { ( } 42 \text { in } \\
\text { intervention) } \\
\text { men and } \\
\text { women }\end{array}$ & $\begin{array}{l}\text { A controlled } \\
\text { study }\end{array}$ & $\begin{array}{l}\text { Intervention group } \\
\text { had less readmissions, } \\
\text { high rates of } \\
\text { absorption into } \\
\text { therapy and } \\
\text { remaining in therapy }\end{array}$ & $\begin{array}{l}\text { Reduce } \\
\text { hospitalisations, } \\
\text { increase compliance } \\
\text { with outpatient } \\
\text { appointments }\end{array}$ \\
\hline 25 & $\begin{array}{l}\text { Kaspow and } \\
\text { Rosenheck } \\
\text { (2007) }\end{array}$ & $\begin{array}{l}\text { USA, } 8 \text { veteran } \\
\text { medical centres }\end{array}$ & $\begin{array}{l}\text { Critical time } \\
\text { intervention } \\
\text { Case management (a } \\
\text { modification of the } \\
\text { critical time } \\
\text { intervention } \\
\text { (CTI) community case } \\
\text { management model) }\end{array}$ & $\begin{array}{l}278 \text { control } \\
\text { cohort, } 206 \\
\text { intervention } \\
\text { cohort }\end{array}$ & $\begin{array}{l}\text { Nonrandomized } \\
\text { pre-post cohort } \\
\text { design }\end{array}$ & $\begin{array}{l}19 \% \text { more days } \\
\text { housed in each } 90-\text { day } \\
\text { reporting period over } \\
\text { the one-year follow- } \\
\text { up and } 14 \% \text { fewer } \\
\text { days in institutional } \\
\text { settings. Veterans } \\
\text { In phase } 2 \text { also had } \\
19 \% \text { lower addiction } \\
\text { severity index alcohol } \\
\text { use scores, } 14 \% \text { lower } \\
\text { drug use scores } \\
\text { And } 8 \% \text { lower } \\
\text { psychiatric problem } \\
\text { scores }\end{array}$ & Reduce homelessness, \\
\hline
\end{tabular}


Table 1 Tabulation and description of the studies used in the systematic review (Continued)

\begin{tabular}{|c|c|c|c|c|c|c|c|}
\hline$\overline{I D}$ & $\begin{array}{l}\text { Authors and } \\
\text { year }\end{array}$ & $\begin{array}{l}\text { Location and } \\
\text { setting }\end{array}$ & Intervention & Participants & Method & Main findings & $\begin{array}{l}\text { Main aim/ problem } \\
\text { to address }\end{array}$ \\
\hline 26 & $\begin{array}{l}\text { Khaleghparast } \\
\text { et al. (2013) }\end{array}$ & Iran, 2 hospitals & $\begin{array}{l}\text { Discharge planning } \\
\text { (self-care training } \\
\text { programme/nursing } \\
\text { process model) }\end{array}$ & $\begin{array}{l}46 \text { service } \\
\text { users }\end{array}$ & $\begin{array}{l}\text { Longitudinal } \\
\text { clinical trial }\end{array}$ & $\begin{array}{l}\text { The intervention } \\
\text { group had improved } \\
\text { clinical symptoms and } \\
\text { higher knowledge } \\
\text { levels compared with } \\
\text { control group. } \\
\text { Statistically } \\
\text { significantly lower } \\
\text { readmissions in the } \\
\text { intervention group. }\end{array}$ & $\begin{array}{l}\text { To increase patient } \\
\text { knowledge, reduce } \\
\text { clinical symptoms and } \\
\text { rehospitalisation. }\end{array}$ \\
\hline 27 & $\begin{array}{l}\text { Khanbhai } \\
\text { et al. (2018) }\end{array}$ & $\begin{array}{l}\text { Australia, } 1 \text { medical } \\
\text { centre }\end{array}$ & Discharge checklist & 230 checklists & $\begin{array}{l}\text { Quasi- } \\
\text { experimental, } \\
\text { pre-post } \\
\text { intervention } \\
\text { design }\end{array}$ & $\begin{array}{l}\text { There was a small, but } \\
\text { statistically non- } \\
\text { significant, reduction } \\
\text { in readmission rates. }\end{array}$ & Reduce readmission \\
\hline 28 & $\begin{array}{l}\text { Kidd et al. } \\
\text { (2016) }\end{array}$ & $\begin{array}{l}\text { Canada, } 1 \text { large } \\
\text { hospital in city }\end{array}$ & $\begin{array}{l}\text { 'Welcome Basket.' (6 } \\
\text { week peer support, } \\
\text { contact on wards, } \\
\text { basket of items, } \\
\text { environmental } \\
\text { support) }\end{array}$ & 23 & $\begin{array}{l}\text { Evaluation- a } \\
\text { mixed methods } \\
\text { design, pre-post } \\
\text { for quantitative } \\
\text { outcomes, inter- } \\
\text { views and re- } \\
\text { admission rates }\end{array}$ & $\begin{array}{l}\text { Pre-post analysis } \\
\text { indicated no change } \\
\text { in psychiatric } \\
\text { symptoms but } \\
\text { improvement in } \\
\text { community } \\
\text { functioning, } \\
\text { community } \\
\text { integration, and } \\
\text { quality of life. No } \\
\text { difference in } \\
\text { readmission }\end{array}$ & $\begin{array}{l}\text { Reduce suboptimal } \\
\text { outcomes in first } \\
\text { month, bridge gap }\end{array}$ \\
\hline 29 & $\begin{array}{l}\text { Kisely et al. } \\
(2017)\end{array}$ & $\begin{array}{l}\text { Australia, } 1 \\
\text { hospital- } \\
\text { intervention and } \\
\text { control wards } \\
\text { within it }\end{array}$ & $\begin{array}{l}\text { Motivational aftercare } \\
\text { planning } \\
\text { (motivational } \\
\text { interviewing with } \\
\text { advance directives) }\end{array}$ & $\begin{array}{l}100 \\
\text { intervention } \\
\text { plans, } 197 \\
\text { control, } 20 \\
\text { service user } \\
\text { interviews }\end{array}$ & $\begin{array}{l}\text { Controlled } \\
\text { before-and-after } \\
\text { design, } \\
\text { interviews }\end{array}$ & $\begin{array}{l}\text { Intervention ward } \\
\text { improved significantly } \\
\text { (e.g. identification of } \\
\text { triggers significantly } \\
\text { increased from } 52 \text { to } \\
94 \% \text {, This did not } \\
\text { occur in the control } \\
\text { wards. Interviews } \\
\text { showed } \\
\text { improvements in } \\
\text { experiences of } \\
\text { discharge planning. }\end{array}$ & $\begin{array}{l}\text { To increase patient } \\
\text { input into discharge } \\
\text { planning, increase } \\
\text { treatment plan } \\
\text { following }\end{array}$ \\
\hline 30 & $\begin{array}{l}\text { Lawn et al. } \\
(2008)\end{array}$ & $\begin{array}{l}\text { Australia, } 3 \\
\text { hospitals }\end{array}$ & Peer support & $\begin{array}{l}\text { No participant } \\
\text { numbers in } \\
\text { evaluation }\end{array}$ & $\begin{array}{l}\text { Evaluation } \\
\text { methodology. }\end{array}$ & $\begin{array}{l}\text { Intervention at this } \\
\text { stage of their recovery } \\
\text { seems highly effective } \\
\text { as an adjunct to } \\
\text { mainstream mental } \\
\text { health services. It has } \\
\text { personal benefit to } \\
\text { consumers and peers, } \\
\text { substantial savings to } \\
\text { systems, as well as } \\
\text { much potential for } \\
\text { encouraging mental } \\
\text { health service culture } \\
\text { and practice towards } \\
\text { a greater recovery } \\
\text { focus and improved } \\
\text { collaboration with GPs }\end{array}$ & $\begin{array}{l}\text { To reduce hospital } \\
\text { avoidance and } \\
\text { facilitate early } \\
\text { discharge }\end{array}$ \\
\hline 31 & $\begin{array}{l}\text { Lin et al. } \\
(2018)\end{array}$ & Taiwan, 1 hospital & $\begin{array}{l}\text { Needs-oriented } \\
\text { hospital discharge } \\
\text { planning for } \\
\text { caregivers }\end{array}$ & $\begin{array}{l}114 \text { caregivers } \\
\text { (of people with } \\
\text { schizophrenia) } \\
57 \text { in each } \\
\text { group }\end{array}$ & $\begin{array}{l}\text { A quasi- } \\
\text { experimental re- } \\
\text { search design }\end{array}$ & $\begin{array}{l}\text { The caregiver burden } \\
\text { and health status of } \\
\text { the experimental } \\
\text { group improved more } \\
\text { significantly compared } \\
\text { with control group. }\end{array}$ & $\begin{array}{l}\text { Reducing readmission } \\
\text { and improving } \\
\text { medication } \\
\text { adherence, reducing } \\
\text { care giver burden }\end{array}$ \\
\hline 32 & $\begin{array}{l}\text { Puschner et al. } \\
\text { (2011) }\end{array}$ & $\begin{array}{l}\text { Germany, } 5 \\
\text { hospitals }\end{array}$ & $\begin{array}{l}\text { Needs-oriented } \\
\text { discharge planning }\end{array}$ & 491 & Multicentre RCT & $\begin{array}{l}\text { No effect of the } \\
\text { intervention on }\end{array}$ & $\begin{array}{l}\text { Reduce high } \\
\text { utilisation of inpatient }\end{array}$ \\
\hline
\end{tabular}


Table 1 Tabulation and description of the studies used in the systematic review (Continued)

\begin{tabular}{|c|c|c|c|c|c|c|c|}
\hline $\mathrm{ID}$ & $\begin{array}{l}\text { Authors and } \\
\text { year }\end{array}$ & $\begin{array}{l}\text { Location and } \\
\text { setting }\end{array}$ & Intervention & Participants & Method & Main findings & $\begin{array}{l}\text { Main aim/ problem } \\
\text { to address }\end{array}$ \\
\hline & & & intervention & & & outcomes. & care \\
\hline 33 & $\begin{array}{l}\text { Reynolds et al. } \\
\text { (2004) }\end{array}$ & $\begin{array}{l}\text { Scotland, } 1 \text { unit, } 3 \\
\text { wards }\end{array}$ & $\begin{array}{l}\text { Transitional Discharge } \\
\text { Model (ward nurse } \\
\text { worked with SU until } \\
\text { relationship built with } \\
\text { community nurse, } \\
\text { then support from } \\
\text { service users) }\end{array}$ & $\begin{array}{l}25 \text { services } \\
\text { user }(14 \\
\text { control, } 11 \\
\text { experimental) }\end{array}$ & $\begin{array}{l}\text { Randomised } \\
\text { experimental } \\
\text { design }\end{array}$ & $\begin{array}{l}\text { Both control and } \\
\text { experimental group } \\
\text { demonstrated } \\
\text { significant } \\
\text { improvements in } \\
\text { symptom severity and } \\
\text { functional ability after } \\
5 \text { months. Usual } \\
\text { treatment subjects in } \\
\text { the control group } \\
\text { were more than twice } \\
\text { as likely to be re- } \\
\text { admitted to hospital. }\end{array}$ & $\begin{array}{l}\text { Readmissions and not } \\
\text { able to adapt to } \\
\text { community, focus on } \\
\text { need for relationships }\end{array}$ \\
\hline 34 & $\begin{array}{l}\text { Rose et al. } \\
\text { (2007) }\end{array}$ & $\begin{array}{l}\text { USA, } 1 \text { large urban } \\
\text { medical centre, } \\
\text { mostly African- } \\
\text { American patients }\end{array}$ & $\begin{array}{l}\text { Transitional care } \\
\text { model a nurse-based } \\
\text { in-home transitional } \\
\text { care intervention }\end{array}$ & $\begin{array}{l}10 \text { service } \\
\text { users } \\
\text { (schizophrenia, } \\
\text { bipolar) }\end{array}$ & $\begin{array}{l}\text { Evaluation- } \\
\text { analysis of nurse } \\
\text { logs }\end{array}$ & $\begin{array}{l}\text { Offers an alternative to } \\
\text { patients who might } \\
\text { otherwise be left } \\
\text { poorly treated or } \\
\text { untreated in the } \\
\text { community setting. }\end{array}$ & $\begin{array}{l}\text { Lack of continuity of } \\
\text { care and meet } \\
\text { immediate post } \\
\text { discharge needs of } \\
\text { SU }\end{array}$ \\
\hline 35 & $\begin{array}{l}\text { Sato et al. } \\
\text { (2012) }\end{array}$ & Japan, 5 hospitals & $\begin{array}{l}\text { Community re-entry } \\
\text { program. Discharge } \\
\text { preparation program } \\
\text { (psychosocial program } \\
\text { for preparing long- } \\
\text { term } \\
\text { hospitalized patients) }\end{array}$ & $\begin{array}{l}26 \\
\text { intervention, } \\
23 \text { control } \\
\text { (schizophrenia) }\end{array}$ & $\mathrm{RCT}$ & $\begin{array}{l}\text { The program may be } \\
\text { capable of promoting } \\
\text { discharge of long- } \\
\text { term hospitalized psy- } \\
\text { chiatric patients. } \\
\text { There was no } \\
\text { significant difference } \\
\text { between both groups } \\
\text { for number of patients } \\
\text { discharged } 6 \text { months } \\
\text { after end of program. }\end{array}$ & $\begin{array}{l}\text { To reduce length of } \\
\text { stay }\end{array}$ \\
\hline 36 & $\begin{array}{l}\text { Scanlan et al. } \\
\text { (2017) }\end{array}$ & $\begin{array}{l}\text { Australia, } 3 \\
\text { geographical areas, } \\
\text { large non- } \\
\text { governmental men- } \\
\text { tal health service }\end{array}$ & $\begin{array}{l}\text { Peer-delivered, } \\
\text { transitional and post- } \\
\text { discharge support } \\
\text { program }\end{array}$ & $\begin{array}{l}38 \text { service } \\
\text { users }\end{array}$ & $\begin{array}{l}\text { Evaluation, } \\
\text { outcome } \\
\text { measures, } \\
\text { interviews }\end{array}$ & $\begin{array}{l}\text { Participants reported } \\
\text { improvements in } \\
\text { functional and clinical } \\
\text { recovery and in the } \\
\text { areas of intellectual, } \\
\text { social and } \\
\text { psychological } \\
\text { wellness. Self-report of } \\
\text { hospital readmissions } \\
\text { suggested that there } \\
\text { was a reduction in } \\
\text { hospital bed days fol- } \\
\text { lowing the program }\end{array}$ & $\begin{array}{l}\text { Reduce readmission, } \\
\text { increase wellbeing }\end{array}$ \\
\hline 37 & $\begin{array}{l}\text { Shaffer et al. } \\
\text { (2015) }\end{array}$ & $\begin{array}{l}\text { USA, } 6 \text { community- } \\
\text { based provider or- } \\
\text { ganizations within } \\
\text { network of a not- } \\
\text { for-profit, managed } \\
\text { behavioural health } \\
\text { care organization }\end{array}$ & $\begin{array}{l}\text { Brief critical time } \\
\text { intervention (a brief, } \\
\text { three-month version } \\
\text { of CTI) }\end{array}$ & $\begin{array}{l}149 \text { adults with } \\
\text { readmission } \\
\text { within } 30 \text { days, } \\
224 \text { control }\end{array}$ & $\begin{array}{l}\text { A quasi- } \\
\text { experimental } \\
\text { investigation }\end{array}$ & $\begin{array}{l}\mathrm{BCTI} \text { was associated } \\
\text { with decreased early } \\
\text { readmission rates, }\end{array}$ & Reduce readmission \\
\hline 38 & $\begin{array}{l}\text { Shaw et al. } \\
(2000)\end{array}$ & $\begin{array}{l}\text { Scotland, } 3 \text { acute } \\
\text { wards, } 1 \text { hospital }\end{array}$ & $\begin{array}{l}\text { Pharmacy discharge } \\
\text { planning (receiving a } \\
\text { baseline } \\
\text { Pharmaceutical needs } \\
\text { assessment, } \\
\text { information about } \\
\text { medicines and then a } \\
\text { Pharmacy discharge } \\
\text { plan sent to their } \\
\text { community } \\
\text { pharmacy) }\end{array}$ & $\begin{array}{l}97 \text { service } \\
\text { users }\end{array}$ & Controlled study & $\begin{array}{l}\text { No significant } \\
\text { difference between } \\
\text { the groups in baseline } \\
\text { medicine knowledge. } \\
\text { One week post- } \\
\text { discharge, both } \\
\text { groups showed } \\
\text { Significant } \\
\text { improvement in } \\
\text { knowledge of } \\
\text { medication from } \\
\text { baseline and was } \\
\text { maintained at } 12\end{array}$ & $\begin{array}{l}\text { To reduce medicine- } \\
\text { related problems that } \\
\text { cause readmission }\end{array}$ \\
\hline
\end{tabular}


Table 1 Tabulation and description of the studies used in the systematic review (Continued)

\begin{tabular}{|c|c|c|c|c|c|c|c|}
\hline $\mathrm{ID}$ & $\begin{array}{l}\text { Authors and } \\
\text { year }\end{array}$ & $\begin{array}{l}\text { Location and } \\
\text { setting }\end{array}$ & Intervention & Participants & Method & Main findings & $\begin{array}{l}\text { Main aim/ problem } \\
\text { to address }\end{array}$ \\
\hline & & & & & & $\begin{array}{l}\text { weeks. } \\
\text { Fewer medication } \\
\text { problems for the } \\
\text { intervention group. }\end{array}$ & \\
\hline 39 & $\begin{array}{l}\text { Simpson et al. } \\
(2014)\end{array}$ & $\begin{array}{l}\text { UK, } 4 \text { wards, inner } \\
\text { city (London) }\end{array}$ & Peer support & $\begin{array}{l}46 \text { service } \\
\text { users } 23 \text { peer } \\
\text { support } 23 \\
\text { control }\end{array}$ & $\begin{array}{l}\text { Pilot } \\
\text { randomised } \\
\text { controlled trial } \\
\text { with economic } \\
\text { evaluation }\end{array}$ & $\begin{array}{l}\text { No statistically } \\
\text { significant benefits for } \\
\text { peer support for hope } \\
\text { or QoL, there is an } \\
\text { indication that hope } \\
\text { may be further } \\
\text { increased in those in } \\
\text { receipt of peer } \\
\text { support. The total cost } \\
\text { per case for the peer } \\
\text { support was } £ 2154 \\
\text { compared to } £ 1922 \\
\text { for control. }\end{array}$ & $\begin{array}{l}\text { To increase hope and } \\
\text { quality of life }\end{array}$ \\
\hline 40 & $\begin{array}{l}\text { Smelson et al. } \\
\text { (2010) }\end{array}$ & $\begin{array}{l}\text { USA, } 1 \text { acute } \\
\text { inpatient } \\
\text { psychiatric unit }\end{array}$ & $\begin{array}{l}\text { Brief Treatment } \\
\text { Engagement ( } 5 \mathrm{~h} \text { per } \\
\text { week of services- } \\
\text { assertive community } \\
\text { treatment using BCTI, } \\
\text { peer support, dual } \\
\text { recovery therapy) }\end{array}$ & $\begin{array}{l}102 \text { veterans, } \\
(56 \text { control })\end{array}$ & $\begin{array}{l}\text { Prospective } \\
\text { randomized trial }\end{array}$ & $\begin{array}{l}69 \% \\
\text { Of intervention } \\
\text { participants attended } \\
\text { an outpatient } \\
\text { appointment within } \\
14 \text { days of discharge, } \\
\text { compared to only } \\
33 \% \text { of control. } \\
\text { Intervention } \\
\text { participants were also } \\
\text { significantly more } \\
\text { likely to be engaged } \\
\text { in outpatient services } \\
\text { at the end of the } \\
\text { intervention period. }\end{array}$ & $\begin{array}{l}\text { Treatment } \\
\text { engagement }\end{array}$ \\
\hline 41 & $\begin{array}{l}\text { Taylor et al. } \\
\text { (2014) }\end{array}$ & $\begin{array}{l}\text { USA, } 1 \text { large } \\
\text { psychiatric hospital }\end{array}$ & $\begin{array}{l}\text { Brief care } \\
\text { management } \\
\text { Intervention (brief } \\
\text { interview prior to } \\
\text { discharge) }\end{array}$ & $\begin{array}{l}87 \\
\text { intervention, } \\
108 \text { control, } \\
195 \text { total }\end{array}$ & Controlled study & $\begin{array}{l}\text { Individuals in the } \\
\text { control group were } \\
\text { more likely to be } \\
\text { readmitted within } 30 \\
\text { days of an index } \\
\text { readmission than } \\
\text { individuals in the } \\
\text { intervention group. }\end{array}$ & $\begin{array}{l}\text { Increase aftercare } \\
\text { engagement, reduce } \\
\text { readmissions }\end{array}$ \\
\hline 42 & $\begin{array}{l}\text { Tomita et al. } \\
\text { (2012) }\end{array}$ & $\begin{array}{l}\text { USA, } 2 \text { New York } \\
\text { City hospitals }\end{array}$ & $\begin{array}{l}\text { Critical time } \\
\text { intervention (CTI) }\end{array}$ & $\begin{array}{l}150 \text { total } \\
\text { previously } \\
\text { homeless men } \\
\text { and women }\end{array}$ & $\mathrm{RCT}$ & $\begin{array}{l}\text { At the end of the } \\
\text { follow-up period, psy- } \\
\text { chiatric re- } \\
\text { hospitalization was } \\
\text { significantly lower for } \\
\text { the group assigned to } \\
\text { CTI compared with } \\
\text { the usual services } \\
\text { group. }\end{array}$ & Reducing readmission \\
\hline 43 & $\begin{array}{l}\text { Virgolesi et al. } \\
\text { (2017) }\end{array}$ & $\begin{array}{l}\text { Italy, } 3 \text { hospitals in } \\
\text { Rome }\end{array}$ & $\begin{array}{l}\text { Nursing discharge } \\
\text { programme (a short- } \\
\text { term nursing dis- } \\
\text { charge programme } \\
\text { with follow-up phone } \\
\text { calls } 7-10 \text { days) }\end{array}$ & 135 patients & $\begin{array}{l}\text { Prospective } \\
\text { correlational } \\
\text { design }\end{array}$ & $\begin{array}{l}\text { The interpersonal and } \\
\text { educational nursing } \\
\text { intervention improves } \\
\text { adherence to a } \\
\text { treatment plan. }\end{array}$ & $\begin{array}{l}\text { Medication adherence } \\
\text { and patient } \\
\text { satisfaction }\end{array}$ \\
\hline 44 & $\begin{array}{l}\text { Walker et al. } \\
(2000)\end{array}$ & $\begin{array}{l}\text { UK, } 3 \text { wards ( } 2 \\
\text { control) }\end{array}$ & $\begin{array}{l}\text { Discharge co- } \\
\text { ordinators }\end{array}$ & $\begin{array}{l}343,119 \\
\text { intervention, } \\
224 \text { control }\end{array}$ & $\begin{array}{l}\text { Controlled } \\
\text { cohort study }\end{array}$ & $\begin{array}{l}\text { No differences in } \\
\text { outcomes } \\
\text { (readmission, LoS, } \\
\text { mental health status, } \\
\text { satisfaction). More } \\
\text { satisfaction for those } \\
\text { without intervention }\end{array}$ & $\begin{array}{l}\text { Improve } \\
\text { communication } \\
\text { between primary and } \\
\text { secondary care }\end{array}$ \\
\hline
\end{tabular}


Table 1 Tabulation and description of the studies used in the systematic review (Continued)

\begin{tabular}{|c|c|c|c|c|c|c|c|}
\hline $\mathrm{ID}$ & $\begin{array}{l}\text { Authors and } \\
\text { year }\end{array}$ & $\begin{array}{l}\text { Location and } \\
\text { setting }\end{array}$ & Intervention & Participants & Method & Main findings & $\begin{array}{l}\text { Main aim/ problem } \\
\text { to address }\end{array}$ \\
\hline 45 & $\begin{array}{l}\text { Zheng and } \\
\text { Arthur (2005) }\end{array}$ & $\begin{array}{l}\text { China, } 1 \text { large } \\
\text { hospital in Beijing }\end{array}$ & Family education & $\begin{array}{l}101 \text { patients } \\
\text { (schizophrenia) }\end{array}$ & $\begin{array}{l}\text { RCT pre-test, } \\
\text { post-test }\end{array}$ & $\begin{array}{l}\text { Significant } \\
\text { improvement in } \\
\text { knowledge about } \\
\text { Schizophrenia in the } \\
\text { experimental group. } \\
\text { Significant difference } \\
\text { in symptom scores } \\
\text { and functioning at } 9 \\
\text { months after } \\
\text { discharge. }\end{array}$ & $\begin{array}{l}\text { Knowledge about } \\
\text { condition and } \\
\text { rehospitalisation. } \\
\text { There is a need for } \\
\text { culturally sensitive } \\
\text { family treatments } \\
\text { offered by nurses }\end{array}$ \\
\hline
\end{tabular}

\section{Critical time intervention}

Critical time intervention (CTI) was the most frequently tested intervention found in this systematic search. CTI primarily aims to reduce homeless, in the 'critical time' following discharge from hospital. It is delivered by trained 'CTI' workers with small caseloads. It is community based and time limited (with gradually reduced contact). It involves 3 phases (1) Transition (2) Try-out (3) Transfer of Care. Five studies [9, 24-26] in the review reported on this intervention; which is primarily aimed at a particular population: those suffering homelessness and acute mental health hospitalisation. All of the studies were conducted in the USA. Two were randomised controlled trials (RCT) of 150 participants [9, 25], two non-randomised experimental design $[24,26]$ and one interviews with staff that deliver CTI $(n=12)$ [27]. Two studies found significant reduction in homelessness for those that received the intervention $[9,24]$. Two studies also aimed to reduce readmission and found significant reductions in readmission rates in the CTI group compared to a control group $[25,26]$. One study employed brief CTI [26]; which delivered the same intervention in a shorter time period. Finally, qualitative interviews found that CTI workers self-identified as 'extra support' and stressed the importance of three dyadic relationships to the success of the intervention: worker-client, workerprimary support and primary-support-client [27]. In summary, the studies reviewed suggest that CTI could be an effective method of reducing homelessness post-discharge and reducing early readmissions.

\section{Transitional discharge model/ transitional relationship model}

The Transitional Discharge Model (TDM) also known as the Transitional Relationship Model, aims to increase continuity of care from hospital to community. Inpatient nurses work with service users until they establish a therapeutic relationship with their community worker. Support from peers (service users living successfully in the community) commencing prior to discharge and for up to 1 year after hospitalisation may be included in this package of support.. Three studies in this review tested this intervention, one was a large scale RCT; which found no significant differences in post-discharge costs or quality of life, but an unexpected finding of early discharge (on average 116 days earlier) [28]. A 25 participant randomised study found a significant reduction in readmissions [29]. The action-oriented research study highlighted the need to address inter-professional team working to improve staff uptake of the intervention [14].

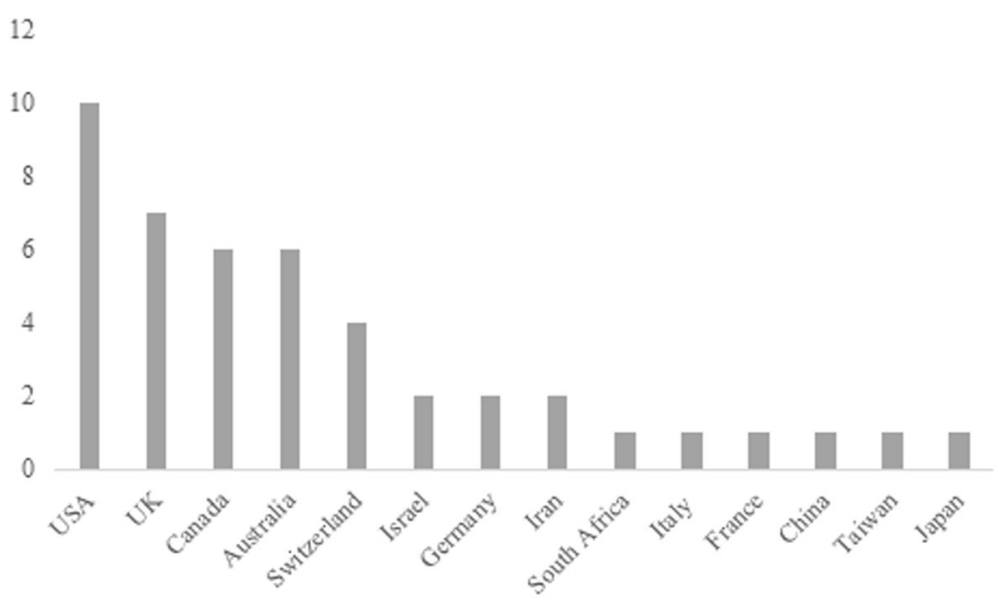

Fig. 2 Countries of origin: The countries of origin of the papers included in the systematic review 


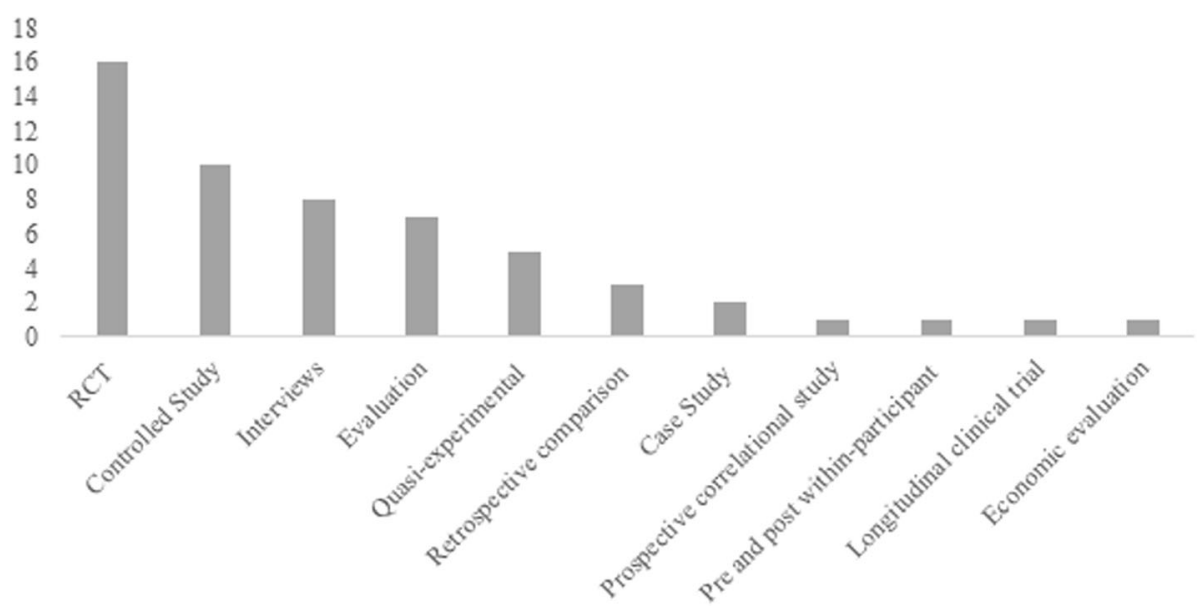

Fig. 3 Design: The design of the papers included in the systematic review

Collectively, the results suggest that the TDM could be effective in reducing readmission and facilitating early discharge.

\section{Peer support}

Peer support is when past service users use their own experiences to help current service users, primarily on a one-to-one basis, but it can exist in various forms. Three studies tested peer support as a distinct single intervention, although others included it as part of multi-component interventions. How peer support was delivered differed across studies, see Table 2 . One evaluation study found a reduction in self-reports of readmission, functional and clinical recovery in 38 service users [30]. Another found benefits to service users and cost reductions for services [31]. However a small pilot RCT found no statistical differences in terms of hope or quality of life [32]. In summary, the studies testing peer support as an individual intervention used small sample sizes and reported heterogeneous outcomes, therefore it's difficult to draw conclusions about its effectiveness.

\section{Contact-based interventions}

The contact-based interventions were grouped together arbitrarily based on the provision of additional postdischarge contact with a professional beyond treatment as usual. Within this group there were various methods of making contact with service users. For example letters, telephone, face-to-face and video. The purpose of contact also varied across studies. Some aimed to reduce suicide, others to improve treatment adherence and one to reduce readmission. There were six contact based intervention studies in this review (see Table 2). Botha et al. aimed to reduce readmissions found no effect of a 90-day transitional care intervention (phone calls and home visits) [33]. Of the studies that aimed to reduce suicide, one found letters to recently discharged service users ineffective [6]. Whilst the other found only very early telephone follow-ups to be effective in a large-scale randomised controlled trial [5]. A small-scale RCT found intensive case management (weekly face-to-face contact and telephone calls) decreased suicidal ideation, increased service contact and satisfaction and improved relationships with professionals [34]. Two studies aimed to use technology to increase treatment adherence, one sent SMS messages and found a significant reduction in readmission and significant difference in treatment utilisation [35]. The other used an Multi-disciplinary team (MDT) videoconference with rural patients and reported greater treatment adherence in the intervention group [36]. In summary, as the interventions varied in terms of delivery and outcomes reported it's difficult to draw conclusions. However, results collectively indicate that speed of follow-up contact is important in terms of suicide prevention and that contactbased interventions may not reduce readmission, but could be a useful mechanism for improving treatment adherence particularly in rural populations.

\section{Role-based interventions}

Role-based interventions were defined by the introduction of a new role, position or job title in addition to treatment as usual. The specific tasks performed by each 'role' varied across the studies, see Table 2 . Seven rolebased intervention studies were included. One study introduced a discharge co-ordinator to improve communication between primary and secondary care but found no significant difference in outcomes (readmission, length of stay, satisfaction, mental health) in a large scale controlled study [37]. Similarly, one large scale RCT found no significant effect of a Post-Discharge Network Co-ordinator on readmission, social support, quality of life or mental health [38]. A case study of 3 patients from the original RCT revealed that this was likely to be due to the small, 
Table 2 Arbitrary clustering of interventions based on intervention categories

\begin{tabular}{|c|c|c|c|}
\hline $\begin{array}{l}\text { Intervention } \\
\text { category }\end{array}$ & $\begin{array}{l}\text { No of } \\
\text { papers }\end{array}$ & Authors name and years & Description of intervention \\
\hline $\begin{array}{l}\text { Critical Time } \\
\text { Intervention }\end{array}$ & 5 & $\begin{array}{l}\text { Tomita (2014), Kasprow (2014), Herman (2011), } \\
\text { Shaffer (2015), Chen (2014) }\end{array}$ & $\begin{array}{l}\text { - Focused on homelessness } \\
\text { - Delivered by CTI workers } \\
\text { - Develop therapeutic relationship } \\
\text { - Time limited (gradually reduced contact), Small caseloads, Community } \\
\text { based } \\
\text { - Phase 1: Transition: Provide support \& begin to connect client to } \\
\text { people and agencies that will assume the primary role of support } \\
\text { - Phase 2: Try-Out: Monitor and strengthen support network and client's } \\
\text { skills. } \\
\text { - Phase 3: Transfer of Care: Terminate CTI services with support network } \\
\text { safely in place. } \\
\text { - Also includes Brief CTI (shorter time) }\end{array}$ \\
\hline $\begin{array}{l}\text { Transitional } \\
\text { Discharge Model } \\
\text { (TDM) }\end{array}$ & 3 & Forchuk (2005), (2012), Reynolds (2004) & $\begin{array}{l}\text { - Ward nurses work with SU until a therapeutic relationship is } \\
\text { established with the community worker } \\
\text { - Then peer support introduced } \\
\text { - AKA/similar to Transitional relationship model (TRM) }\end{array}$ \\
\hline
\end{tabular}

Transitional care 2 Hanrahan (2014), Rose (2007)
model
model Exbrayat (2017), De Leo (2007), Botha (2018)

Walker (2000), Virgolesi (2017), Hengartner (2016,17), Jenson (2010), Bonsack (2016), Hampson (2000)
- Nurse based in home transitional care intervention, to increase CoC

- A) comprehensive discharge planning

- B) home visits and telephone contacts with a nurse (assessments, care, psychoeducation)

- Aimed at most challenging patients with long history of readmission

- Immediately providing intensive support and identifying problems early before readmission

- Increase QoL through symptom management, medication adherence and enhanced family support

- Scanlan: Peer-delivered support programme: peers delivering providing individualised practical and emotional support to individuals for 6-8 weeks following discharge

- Simpson: Peer support workers to provide peer support for 4 weeks to discharged service users, initial contact begins on ward

- Lawn: Peer support workers trained alongside other health professionals. Service users matched to peers experience and skills, 8-12 h, 1-2 week period. Also hospital avoidance packages for those who are thought to need them.

- Bennewith: Letters sent to follow up recently discharged service users at home

- Bauer: SMS sent to recently discharged service users about maintaining treatment

- D'Souza: MDT videoconferencing with rural patients post-discharge

- Exbrayat: Nurse telephone follow up 8, 30 and 60 days post suicide attempt

- De Leo: Intensive case management - Weekly face-to-face contact with community case manager and telephone calls from counsellors

- Botha: 90 Day Transitional care intervention - 4 phone calls, 1 home visit focusing on maintaining adherence, appointment reminders and psychoeducation

- Walker: Discharge co-ordinators - educational role with service users and family, develop relationships, 6-8 weeks post-discharge, Dr. routinely telephoning GP practice regarding impending discharge and arrange an appointment with GP within 7 days of discharge, posting discharge summary to practice

- Virgolesi: Nursing discharge programme- information interventions provided by nursing staff, direct hospital medication, distribution and follow-up telephone calls. Nurses attend a 1-h class organised into 5 modules: introduction to medication adherence, conceptual framework of medication adherence, medication adherence, intervention programmes, structure of medication adherence interview, and case studies.

- Hengartner: Post-discharge network co-ordinator - delivered by social workers, support service users to build and maintain social network and link to community care system - goes to ward on 1st week, develops plan before discharge, home visit 3 days post discharge

- Jenson: Community based discharge planning - in reach, community nurse visits ward daily 
Table 2 Arbitrary clustering of interventions based on intervention categories (Continued)

Intervention No of Authors name and years Description of intervention

category papers

Pharmacist 2 Abraham (2017), Shaw (2000)

Interventions

Intervention to

Forchuk 2008, 2013

prevent

homelessness

(Psycho)

educational

Kariel-Lauer (2000), Zheng (2005), Sato (2012), Khaleghparast (2013), Hegadus (2018)

Needs-oriented

discharge

planning

Puschner (2011), Lin (2018)

Whole Care

Pathway

Initiatives
- Bonsack: Transitional case management -- a nurse, or a social worker was added to the treatment as usual procedure. Their role was not to replace the other care providers but to coordinate care provision and to represent the patient's viewpoint.

- Hampson: Community link team - to facilitate early discharge teambased service offering intensive support during the day.

- Abraham: Pharmacist consult intervention- psychiatrist has to order a pharmacist consult in the EHR for all LAI orders, hard copy of form sent to inpatient pharmacy and clinical pharmacist. Pharmacist has to approve LAl prescription before administered. Day of discharge injection clinic. Pharmacist led transitions in care program and medication delivery available prior to discharge. Following discharge continued treatment in outpatient clinic.

- Shaw: Pharmacy discharge planning- baseline pharma needs assessment, information about medicines and then plan sent to community pharmacist

- Immediate assistance in accessing housing, assistance paying first month rent

- Kariel-Lauer: Re-entry group - short term group meetings, psychoeducational approach

- Zheng: Family education- $8 \mathrm{~h}$ with service users, $36 \mathrm{~h}$ with family in hospital, $2 \mathrm{~h}$ per month for $3 \mathrm{~m}$ post-discharge. Nurse with $>10 \mathrm{yr}$ experience provided intervention. Purpose is to educate families about schizophrenia, treatment, teach skills to help families cope

- Sato: Community re-entry program- discharge preparation programme - psychosocial preparation for long-term service users

- Khaleghparast: Self-care training programme, delivered by nurses- 61 h sessions pre-discharge, 1 a fortnight post-discharge

- Hegedus: Short transitional intervention in psychiatry - aims primarily to prepare patients for specific situations that could arise during the days immediately following discharge- cards with potential scenarios on

- Puschner: Manualised needs led discharge planning and monitoring intervention with 2 intertwined sessions, 1 at discharge 13 months after. The intervention aimed at improving this communication (between primary and secondary) by means of information (needs assessment)-based standardised recommendations for outpatient treatment and monitoring of compliance with these recommendations.

- Lin: Needs-orientated discharge planning for caregivers- nurses served as care coordinators and provided 6-step hospital discharge planning services to caregivers. Integrated therapeutic partnership, mental health education, and needs oriented services.

- Attfield: Integrated care pathways- reducing unnecessary tests interventions and duplications- (ICPS), is a 'multidisciplinary plan of care that provides detailed guidance for each stage in the care of a patient with a specific condition, over a given period of time'

- Juven-Wetzler: Continuation of care model- continuation of care by the same staff from the ward rather than outpatient referral

- Kidd: Welcome Basket- 6 weeks- peer support- contact on wards prior to discharge and post, basket of items, environmental support

- Smelson: Brief treatment engagement- $5 \mathrm{~h}$ per week of services in community- assertive community treatment using BCTI, peer support, dual recovery therapy

- Ghadiri: Aftercare Services- 3 components, follow-up care (home visits or telephone), family psychoeducation, social skills training for patients

- Doctor's checklist as an aid memoir 
Table 2 Arbitrary clustering of interventions based on intervention categories (Continued)

\begin{tabular}{llll}
\hline $\begin{array}{l}\text { Intervention } \\
\text { category }\end{array}$ & $\begin{array}{l}\text { No of } \\
\text { papers }\end{array}$ & Authors name and years & Description of intervention \\
\hline $\begin{array}{l}\text { Motivational } \\
\text { Aftercare }\end{array}$ & 1 & Kisely (2017) & • Motivational interviewing with advanced directives \\
$\begin{array}{l}\text { Planning } \\
\text { Brief Care }\end{array}$ & 1 & Taylor (2014) & $\begin{array}{l}\text { - A brief interview that addresses goals and barriers to treatment which } \\
\text { was administered by care managers of a managed behavioural health } \\
\text { organization prior to the individuals' discharge }\end{array}$ \\
\hline
\end{tabular}

conflictual or unstable social networks of service users [39]. Two roles (community based discharge team and community links team) that focused on bridging the gap between inpatient and community care were found to be effective in either reducing readmission rates [40] or median length of stay [41]. The introduction of a case management role was found not to reduce readmissions [42]. However, a nursing discharge programme was found to improve medication adherence [43]. In summary, rolebased interventions are introduced to address different aspects of the discharge process and, therefore comparison is difficult. However, all bar one of the role-based interventions, had no effect on readmission rates. This could indicate that readmission rates are not influenced by the introduction of new staff roles.

\section{Educational interventions}

Educational interventions, focused on the delivery of training or education to service users or their families. Five educational interventions were included in the review, four of which were conducted in Asia. Four focus primarily on teaching various self-management techniques to service users, whilst one focuses on educating family members too. As these were educational interventions, the outcomes measured frequently concerned knowledge levels. Two studies reported a significant increase in knowledge about the psychological condition post intervention $[44,45]$. Others aimed to use education to reduce readmission, two reported this effect $[45,46]$, whilst one reported no effect [47]. Educational interventions also showed some effect on reduction of symptoms, and treatment adherence [44-46]. One intervention taught service users how to cope and manage situations that may occur in the community. However no significant results were reported [48]. In summary, the outcomes measured in the educational interventions differ from those in the other interventions, with a greater focus on knowledge and behavioural outcomes, whilst there is an indication that psychoeducational interventions increase knowledge about ones condition, there is evidence to suggest that educational interventions may also improve some service-level outcomes such as readmission. However, the one study that reports behavioural/emotional outcomes reported no effect [48].

\section{Other interventions}

There were some interventions included in the review that did not fit within the aforementioned primary categories, these groups had 3 or fewer studies, see Table 2. These were categorised as pharmacy interventions [11, 12] (medications focused interventions led by pharmacists), needs-orientated discharge planning [44, 49] (discharge planning interventions led by the needs of individuals), intervention to prevent homelessness (an intervention developed by Forchuk et al. [10, 50] focused only on homeless individuals), transitional care model (a nurse-based in home initiative) [51, 52], whole care pathway initiatives $[53,54]$ (that consider multiple agencies in the care pathway) and multi-component models [55-57] (using multiple interventions simultaneously). Despite studies reporting on these interventions they tended to be single instances and do not provide sufficient evidence for narrative synthesis on a categorical level.

\section{Variability of outcomes}

Due to the vast differences in study design and populations, the outcomes measured varied considerably. Outcome was defined broadly when extracting the data to include anything that was measured or reported as a result of the intervention. Due to the differences in design, only RCTs reported primary and secondary clinical outcomes. Using this outcome definition, there were 69 unique outcomes reported across the studies. Whilst there were commonalities amongst some (readmission, length of stay, symptoms), many looked at specific outcomes in regards to a particular research question (addiction severity, concern about discharge, financial cost to system). Even those studies that reported the same outcomes measured them in different ways. For example, readmission was measured using various time frames, e.g. within a week, within 30 days, within a year. In addition, data were collected in various ways, e.g. interviews with service users or collecting hospital data. Table 3 shows the most commonly reported outcomes and the number of studies that reported each particular outcome.

\section{Theoretical assumptions}

In order to consider the effectiveness of the interventions presented in this review, we need to first understand the 
underpinning theoretical assumptions of each intervention. Theory of change is not explicitly used in any of the reviewed studies, and many of the assumptions about the challenges associated with discharge are implicit within the design and evaluation of the intervention.

During the process of narrative synthesis, studies were clustered and grouped in multiple ways, one such way exemplified the threats to safety that the intervention aimed to solve and whether an effect was subsequently reported. As the outcomes were heterogeneous, it was difficult to directly compare outcomes, so instead we grouped the interventions in terms of the safety challenges they aimed to address, either implicitly or explicitly (see Additional file 5).

\section{Reducing readmission}

The most common challenge that the interventions aimed to solve was readmission to an acute ward within a given short-term period, sometimes indicative of shortcomings in service provision $[6,12,18,25,26,28,33$, $36-38,40,41,53,54,58-60]$. Whilst some studies found evidence for a reduction in readmission due to the interventions $[26,28,40]$, many failed to evidence this effect $[6,33,36]$. The studies that had an effect on readmission tended to focus on either education, therapeutic relationships or increased continuity of care [28, 61, 62]. Many of the successful interventions bridged the boundaries between ward and community by providing care from ward based professionals in the community $[28,54]$ or having community teams leading discharge planning on the wards [40]. Interventions that were successful in reducing readmission (primarily in Asian countries) had a psychoeducational focus $[45,46]$. Other effective interventions were focused on a particular population, for example

Table 3 Number of studies that reported the most common outcomes

\begin{tabular}{ll}
\hline Outcome & No of Studies \\
\hline Readmission & 22 \\
Length of stay & 11 \\
Mental health symptoms/psychopathology & 10 \\
Quality of life & 7 \\
Treatment adherence & 5 \\
Outpatient/appointment adherence & 4 \\
In housing & 4 \\
Global functioning & 4 \\
Service user satisfaction with discharge & 4 \\
Medication adherence & 3 \\
Depression & 3 \\
Knowledge about own condition & 3 \\
Service user satisfaction with treatment & 3 \\
\hline
\end{tabular}

homeless individuals and managing financial/environmental challenges that these particular service users faced $[25,26]$.

More interventions were shown to have little effect on readmission than those that did. Some of these interventions shared commonalities with the successful interventions, for example the community link team, offering intensive support in the community during the day [41]. Contact-based interventions were particularly unsuccessful in terms of reducing readmission, for example videoconferencing [36], follow-up letters [6] and follow-up phone calls [33]. A few studies that considered care pathways from a staff-driven or service-level perspective were also not shown to be effective in terms of reducing readmissions [53, 58].

Many of the interventions that failed to reduce readmission, were arbitrarily categorised as role-based, e.g. psychiatric discharge co-ordinators [37], pharmacy discharge planners [12], community link team [41], postdischarge network co-ordinators [38]. This suggests that it may not be sufficient to introduce a new role as a single intervention. There is also evidence to suggest that care co-ordinating roles may result in high levels of stress and burnout [59]. Furthermore, issues that might lead to readmission are manifest across multiple dimensions, for example clinical, personal or social., Researchers are also increasingly questioning the validity of readmission outcome data, as better hospitals keep people alive, safe and cared for therefore multiple readmissions are in some cases an indication that a hospital is safer $[18,60]$.

A number of the interventions in this review that span boundaries have proven successful in terms of reducing readmission. There is also success in the psychoeducational interventions and those that focus on therapeutic relationships, indicating that tackling the personal and emotional elements of the care transition may be equally important when aiming to reduce psychiatric readmission. Whilst it is difficult to make any conclusions about effectiveness of interventions when the outcomes and geographical context are heterogeneous, the most promising results in terms of readmission involve reducing the epistemic, professional and physical boundaries between hospital and community. Therefore encouraging therapeutic relationships, education and empowerment of service users.

\section{Improving wellbeing and/or reducing symptoms}

Many of the interventions that focused on the care transition inherently aim to improve wellbeing and reduce symptoms $[6,29,35,55]$. The studies that report evidence of this have few commonalities, see Additional file 5. Whilst some contact based interventions show an effect [35] others show no effect [6]. Some interventions show an increase in quality of life but no change in 
symptoms [55], on the contrary, others report a reduction of symptoms but no increase in quality of life [29]. Perhaps this lack of clarity in the results could be a manifestation of using such subjective, difficult to measure outcomes that could be easily confounded by factors peripheral to the discharge intervention, rather than the effect of the transition intervention. Many of the interventions that focused on the transition between inpatient and community care inherently aimed to improve wellbeing and reduce symptoms. The studies that report evidence of this have few commonalities, see Additional file 5. Whilst some contact based interventions show an effect [35] others show none [6]. Some interventions show an increase in quality of life but no change in symptoms [55]. On the contrary, others report a reduction of symptoms but no increase in quality of life [29]. Perhaps this lack of clarity in the results could be a manifestation of using such subjective, difficult to measure outcomes that could easily be confounded by factors peripheral to the discharge intervention.

\section{Improving treatment and/or medication adherence}

A number of studies aimed to improve treatment adherence $[12,35,36,40,43,50,52,56]$. The few interventions that report success in improving treatment or medication adherence tend to be brief $[50,56]$, involve a co-ordinating agent $[12,43,50]$ or use technology enhanced contact methods [35, 40]. Unlike readmission, the successful interventions that aim to increase treatment adherence tend to be role-based and some included a co-ordinating agent either a nurse $[43,52]$ or a pharmacist [12]. Similarly, whilst contact based interventions were less effective in reducing readmission, two contact based interventions improved treatment adherence $[35,36]$.

\section{Reducing homelessness}

There were two interventions included in this review that focused on a sub-population within an acute ward, homeless individuals $[10,25-27,63]$. All of the interventions reported success in reducing homelessness [10, 25-27, 63]. The interventions studied by Forchuk and colleagues looked at financial assistance and support in accessing housing, essentially providing resources that service users might not otherwise have [10,63]. Whereas the other intervention, CTI, focused on therapeutic relationships, and helping service users access services [2527]. Both interventions focused primarily on homelessness but also reported benefits in terms of other outcomes like readmission.

\section{Reducing suicide}

Only three studies in the review focused on reducing suicide post-discharge $[5,6,34]$. In the unsuccessful intervention 8 letters were sent to service users in the year after discharge, but this had no effect on suicide [6]. The two effective interventions focused either on early follow up post-discharge either by telephone [5] or consistent weekly face-to-face contact [34]. Indicating the immediacy and consistency may be key to interventions looking to solve this problem. This highlights that continuity of care with a human is more effective than indirect communication via letters as a means of reducing suicide post-discharge.

\section{Accelerating discharge}

The interventions that are successful in reducing length of stay and accelerating discharge tend to have a systems or process level focus. For example integrated care pathways are evidenced to facilitate early discharge [53], as is introducing a community link team [41]. Whereas interventions that focus on a single element of the care pathway, or a single person seem less successful in solving this problem, for example the introduction of a team to co-ordinate discharge planning had little effect on length of stay [61, 64]. Similarly, introducing a single professional to address this challenge [37] or relying on the education of the service user [47], also proved unsuccessful. This could indicate that individual agents i.e. a single professional, team or service-user, are often disempowered within a multi-agency system, and therefore unable to generate meaningful change.

\section{Examination of facilitators and challenges/barriers}

There were commonalities within the studies in terms of barriers and facilitators of effective implementation of interventions. All of which could be categorised as either staff level, service level, or service-user level, many sit within multiple categories. From a service level perspective, barriers were related to insufficient funding of services or interventions, ineffective information sharing and the effect of the physical location of services, particularly for rural community services $[10,12,26,50]$. The structural effect was also a reported facilitator along with planning [65]. There were commonalities within the studies in terms of barriers and facilitators of effective implementation of interventions. All of which could be categorised as either staff level, service level, or service-user level and many sit within multiple categories. From a service level perspective, barriers were related to insufficient funding of services or interventions, ineffective information sharing and the effect of the physical location of services, particularly for rural community services $[10,12,26,61]$. Effective planning was also a reported facilitator [64].

The effectiveness of an intervention was often highly dependent on the behaviours, opinions, affect and education of the staff delivering them. The willingness of 
staff to adapt and exhibit flexibility was key, as was providing staff with adequate training around the intervention [14, 28]. If staff worked in a multi-disciplinary manner, this was also considered facilitative to some interventions [52]. Having a 'champion' or staff member that advocates for the intervention was facilitative [14]. Staff were more responsive to interventions that reduced their workload or stress, as opposed to increasing it [14, $40,41]$. One staff level barrier was a lack of behaviour change in response to the intervention [28].

Barriers to successful intervention that were reported at a service-user level included behaviours that are often in opposition to recovery, for example substance-misuse, dependency on services or unstable social relationships $[33,39,41]$. Similarly, facilitators on a service-user level included behaviours or affect that are facilitative of recovery, such as a sense of belonging within community or community services, structured daily routine within the community and being a part of stable and structured social networks [30, 38, 40, 46, 51]. This does not indicate that the success of the intervention is dependent on the behaviour of the service users, but instead highlights the considerable effect of the complicated personal and social variables that surround mental health care transition interventions.

A number of facilitators and barriers transposed these distinct categories. From a barriers perspective, miscommunication, a lack of shared knowledge or accountability could be pejorative to intervention effectiveness on either a service, staff or service-user level or between groups [14]. Similarly, from a facilitator perspective comparable themes transcend the groups: communication and shared decision making within and between the groups was a key facilitator [52]. Connection amongst providers i.e. a supportive information sharing system, was also facilitative amongst staff groups and service providers [10]. Similarly, therapeutic relationships between staff and service users were considered facilitative [9, 27, 34]. Additional files 6 and 7 show visual depictions of barriers and facilitators.

\section{Assessing robustness}

We used the Mixed Methods Appraisal Tool (MMAT) [20] to assess the quality of the studies included in the systematic review. It must be noted that a number of studies in this review had less than 20 participants and take an evaluative or pilot approach rather than a quantitative robust test of effectiveness. Additional file 3 outlines the quality assessment of each study. All of the studies included in the review met the screening criteria (used as a measure of minimum quality). The tool does not suggest researchers score the papers, however the table highlights the differences in study quality. As this systematic review does not make recommendations for the most effective intervention, the robustness of the studies is of lesser concern. Interestingly, many of the pilot studies found no or little effect of the interventions and it seems such interventions are rarely re-tested. For example, to our knowledge Walker et al., [37] is the only study to specifically test 'discharge co-ordinators' in relation to mental health, despite evidence that this method is effective in other patient populations and its inclusion in the UK National Institute for Health and Care Excellence (NICE) guideline regarding transitional care [66]. Some studies use a similar approach, such as care managers responsible for facilitating discharge [50], but the name 'discharge co-ordinator' has not been tested to our knowledge since. Hence, assumptions about the effectiveness of any particular intervention in this review are often based on the results of a small number of studies.

\section{Discussion}

The studies included in the review are varied in terms of origin and design. Whilst this review uses a broad inclusion criteria to demonstrate the variability in challenges they aim to address, this means that there is variability of the baseline health systems in which the intervention is implemented, complicating comparison. For example, the interventions from Asia frequently reported higher effectiveness rates than those in the UK or USA, indicating that there are potentially cultural differences within complex systems that affect comparability of outcomes cross-culturally. This may include differences in the baseline or treatment as usual conditions. In the UK, for example, NICE guidelines recommend elements of some interventions as standard practice, (i.e. having a named staff member manage this discharge) [67]. Therefore, any effects of such interventions in the UK could be diminished within standard clinical practice.

This review highlights the different approaches that have been used internationally to tackle the varied challenges that discharge from an acute, inpatient mental health care poses. The variability of the interventions and the outcomes are likely to be a manifestation of the variation in how each research team interprets the problems associated with discharge. For example, those interventions that focus on pharmacist involvement, consider the active risk factor of medication non-adherence, whilst the contact-based, or whole system interventions articulate the problem of ineffective communication. Some researchers chose to only measure outcomes relevant to the specific problem they aim to address, whilst others do not articulate how the measures used indicate an improvement. Understanding the effective elements of interventions that address specific problems, would have greater advantages for healthcare professionals looking to improve practice or policy makers attempting to improve quality and safety at a service-level. 
The interventions reviewed are spread across a spectrum ranging from addressing a single problem within a single agency with a single solution, to multiple solutions addressing multi-agency problems. Within which some interventions include multiple elements, i.e. 1) peer support 2) group meetings and 3) therapeutic relationship building. The notion that one intervention can solve a multitude of safety threats is also not evidenced in the wider care transitions literature $[68,67]$. Hence, it's difficult to assess the effect of each component of multi-stage interventions on each single problem, particularly without an explicit underlying theory of change.

Designated roles supporting the transition of service users from inpatient to community care was highlighted in a number studies included in this review. Care coordination has a long history within mental health services, for example in England and Wales the introduction of care co-ordinators stems from the Care Programme Approach in 1990 [69]. Assigned as the main point of contact for service users, care co-ordinators should facilitate care across agencies for an individual, including the transition from hospital to community. Care co-ordination is also an emergent concept in other areas of healthcare, particularly where individuals have complex needs. Whilst studies suggest that professionals working in co-ordination roles have high job satisfaction, they also experience high levels of stress and burnout $[59,70]$. Therefore, when implementing new transitional roles consideration needs to be given to how they will fit with existing co-ordination roles and the support required by the individual undertaken them.

The heterogeneity in terms of outcome reporting made meaningful comparison of any interventions difficult. Even when interventions focused on a single solution, researchers measured select outcomes using different measurement tools. It is difficult therefore to assess the effectiveness of any single element with regards to any single outcome measure. This is in line with a systematic review of interventions that aimed to reduce readmission, whereby quantitative meta-synthesis could not be conducted [18]. This could also be exacerbated by the fact that the outcomes are arguably not indicative of the success of an intervention as they can be easily confounded by external variables. A recent report by The Kings Fund has questioned the validity of using clinical outcomes for a mental health population and recognised the importance of social and emotional outcomes [71].

Very few of the papers were explicit about the underlying theory of change. They often had unclear assumptions about what the nature of the problem was and how the interventions aimed to address it. This was further informed by the selection of the outcomes or measures used; which seemed in some cases to be pragmatic proxies rather than based upon a specific theory of change. For example, there is an emerging body of literature questioning the effectiveness of readmission as an outcome in mental health, as it only describes service use not clinical need [19, 72]. Research suggests that using a framework to guide improvement initiatives is beneficial, for example using the action-effect method (a systematic, structured approach to identify and articulate an improvement interventions theoretical assumption) [73].

In this paper we present a review of various discharge interventions that are not explicitly patient safety interventions, but that focus on improving quality and safety by addressing risk factors such as ineffective continuity of care or communication. All of the interventions aim to improve quality and safety, but are based upon limited understanding or articulation of what the quality and safety elements of healthcare are, nor are they informed by the safety literature. For example, they do not engage with patient safety literature that describes active and latent risk factors [74, 75], nor the literature around 'systems-thinking' approaches to managing risk [75]. Current thinking in the field of patient safety emphasises the contribution of upstream 'latent factors' in conditioning, exacerbating and enabling 'active errors' or mistakes in the organisation and delivery of care $[74,75]$. These often involve local workplace and environmental factors, management pressures and organisational cultures. Such system factors are described as heightened at the point of discharge because care transitions tend to involve multiple sets of system factors interacting in the form of a complex system, as the patient moves across care domains [76]. Many of the threats to safety that are present in this time period are not explicit or directly visible in the working environment, as noted above they can be seen as latent risk factors whose impact on the continuity, quality and safety of care can be difficult to detect [74]. In relation to mental health care transitions, literature has outlined multiple systems-level risk factors in this time period, namely the lack of continuity of care and difficulties with communication between organisations and professionals [77-79] and many of the interventions implicitly or explicitly aimed to address one or more of these.

There are few unambiguous and conclusive findings from this review in terms of the effectiveness of interventions in addressing the distinct problems associated with discharge from acute mental healthcare settings, which is similar to other systematic reviews in this field $[18,72]$. The synthesis suggests that the interventions that aim to reduce homelessness are generally effective $[24,26,27,63]$. In particular, the review finds that these successful interventions either provide resources or psychosocial and/or therapeutic support in securing accommodation. This arguably indicates the importance of addressing a single risk factor with a single solution, ideally with an underpinning explicit theory of change. 
Similarly, with interventions that aim to improve treatment adherence, there seems to be some success in introducing a co-ordinating agent (assigned nurse, social worker or pharmacist) $[12,43,50,52]$ or technology enhanced contact methods [34, 35].

When considering the reduction of readmission, the most successful interventions aim to bridge the epistemic, professional and physical boundaries between hospital and community [14, 29, 61], either locating community staff on the ward, or ward staff in the community, increasing continuity of care or increasing knowledge of service users and families (see also [68]) [67].). Some examples of this are the Community-Based Discharge Planning [40] and the Transitional Discharge Model [29]. The commonality amongst interventions that successfully accelerated discharge is the use of a multi-agency, systems level approach to intervention [28, $41,53]$, suggesting a systems-level approach is more successful than a single intervention (such as a new role) in accelerating discharge.

When considering the different types of interventions, there is some evidence that CTI is an effective method of reducing post-discharge homelessness. There is some evidence that the Transitional Discharge Model reduced readmission and facilitated earlier discharge. When peer support is used as a single intervention, there is very little evidence for effectiveness, this is probably due to the small evidence base of three studies and no effects reported in the only RCT. However, peer support has been used effectively as a component of a wider intervention that looks to increase continuity of care. Contact interventions varied in terms of format and scope in this review, however when addressing post-discharge suicide there some evidence that very early human contact could be effective. There is little evidence to suggest that contact-based interventions reduce readmissions, but some evidence for improvements in treatment adherence, particularly in rural populations. Similarly, rolebased interventions were ineffective in reducing readmissions in all but one study, there is some evidence that introducing the correct roles could increase treatment adherence or reduce length of stay. The difference in reported success is likely due to the purpose of the roles. Roles that were introduced to address social/environmental factors were less successful than clinically focussed ones that aimed to increase treatment adherence. Boundary spanning roles (bridging hospital and the community) were also particularly successful. When considering the effect of interventions on readmission, it is important to re-consider the aforementioned emerging literature arguing against the effectiveness of readmission as outcome [19, 72].

Educational interventions seem highly successful in increasing knowledge outcomes in both service-users and care-givers. This increase in knowledge is also associated with subsequent effects in clinical and system-level outcomes such as readmission, symptom reduction and treatment adherence. The educational interventions predominantly originated from Asia and were uncommon in Europe and North America. However, the results could suggest that empowering service users and families/carers with knowledge rather than intervening only with staff and systems could be beneficial, beyond increased knowledge.

Overall the most effective interventions focus on addressing a single problem, they express explicitly the problem they aim to address and how success with be indicated by measurements. This is evident in the interventions that aim to address homelessness or increase knowledge of one's condition. This could indicate that the precise specification of the intervention may be less important than how the intervention develops a better understanding of the problem, and hence uses a theory of change.

\section{Recommendations}

In summary, to allow for a greater understanding of the elements of interventions that effectively reduce risk factors, a more structured approach to testing interventions is needed. This could be operationalised in multiple ways. First, by generating an agreed upon core outcome set to be used as standard in all future mental health discharge interventions (any unique outcomes would be used in addition to this). Second, more clarity is need in explicitly stating the problem (or latent risk factor) that an intervention aims to address (or each element in a multi-component intervention). This could explain or reduce the variability of effectiveness between similar interventions by providing more structure, transparency and means of comparison and subsequently advancement. In line with the majority of implementation research findings, the reviewed papers have very little underpinning theory (more specifically, theory of change) and articulation of what is needed within a complex system for the intervention to be successful [80]. Conceptualising these problems from a patient safety, systems-thinking perspective and with an explicit theory of change may make it easier to: 1) describe the specific problem the interventions aim to address; 2) understand the elements of an intervention that are effective to produce the desired intermediate or long term outcomes and c) understand what long term outcomes would indicate an effective intervention.

\section{Limitations}

By utilising a less restrictive search strategy the outcomes reported are broad, and the aim of the studies varied. Thus making quantitative comparison difficult. Due to variances in outcomes reported, the quality of the studies 
used, cultural differences and the small number of within each intervention category no conclusive evidence can be drawn with regards to the effectiveness of any particular intervention. In an attempt to highlight and synthesise a breadth of interventions, the studies included in the review were not excluded due to risk of bias or quality, provided they met the basic screening questions in the MMAT. Due to budgetary constraints this review excluded papers that were not published in English and we acknowledge that this may have had an effect on the results of the synthesis and resulted in inclusion of papers that primarily represent English speaking cultures.

This review did not highlight sub-acute service models such as step-up or step-down services; which are increasingly common in the literature [86]. These are not specifically discharge interventions, but instead another service that often occupies the transitional gap. Future research should review the effects of sub-acute services in comparison to 'discharge interventions'.

\section{Conclusions}

There are numerous risk factors present in the chaotic, emotionally-charged period of discharge from an acute inpatient mental health ward. Heterogeneous interventions have been developed internationally in an attempt to solve some of these problems with variable success. Improving homogeneity of outcome reporting and applying theory of change to future research would allow better comparison of interventions.

\section{Supplementary information}

Supplementary information accompanies this paper at https://doi.org/10. 1186/s12913-019-4658-0.

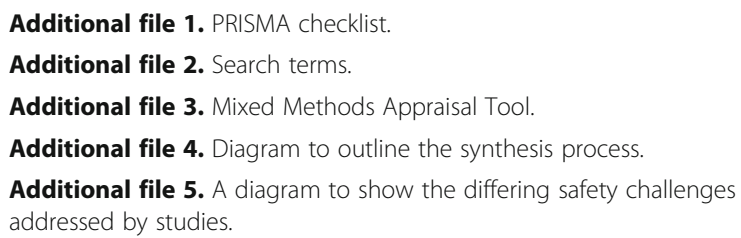

\section{Abbreviations}

BCTI: Brief Critical Time Intervention; CLT: Community Link Team; CoC: Continuity of Care; CTI: Critical Time Intervention; GP: General Practitioner; ICP: Integrated Care Pathway; LAl: Long Acting Injectable; LoS: Length of Stay; MDT: Multi-disciplinary team; MMAT: Mixed Methods Appraisal Tool; NHS: National Health Service; NICE: National Institute for Health and Care Excellence; QoL: Quality of Life; RCT: Randomised Controlled Trial; SMI: Serious Mental IIIness; SMS: Short message service; SU: Service User; TAU: Treatment as Usual; TCM: Transitional Care Model; TDM: Transitional Discharge Model; TRM: Transitional Relationship Model; UK: United Kingdom; USA: United States of America

Acknowledgements Not Applicable.

\section{Authors' contributions}

NT prepared the manuscript, screened and extracted data from each paper, conducted the narrative synthesis. NW and JW conceived the design of study, screened and extracted data from a proportion of papers and contributed to the preparation of the manuscript. All authors approved the final manuscript.

\section{Funding}

This work was funded by the National Institute for Health Research (NIHR) Greater Manchester Patient Safety Translational Research Centre (NIHR Greater Manchester PSTRC). The views expressed are those of the author(s) and not necessarily those of the NIHR or the Department of Health and Social Care.

\section{Availability of data and materials}

The datasets used and/or analysed during the current study are available from the corresponding author on reasonable request.

\section{Ethics approval and consent to participate}

Not Applicable.

\section{Consent for publication}

Not Applicable.

\section{Competing interests}

The authors declare that they have no competing interests.

\section{Author details}

${ }^{1}$ NIHR Greater Manchester Patient Safety Translational Research Centre, The University of Manchester, Manchester, UK. ${ }^{2}$ School of Health Sciences, University of Nottingham, Nottingham, UK. ${ }^{3}$ Health Services Management Centre, School of Social Policy, University of Birmingham, Birmingham, UK.

Received: 6 June 2019 Accepted: 20 October 2019

Published online: 25 November 2019

\section{References}

1. Wright N, Rowley E, Chopra A, Gregoriou K, Waring J. From admission to discharge in mental health services: a qualitative analysis of service user involvement. Health Expect. 2016;19:367-76. https://doi.org/10.1111/hex. 12361.

2. Beresford P, Wallcraft J. Psychiatric system survivors and emancipatory research: issues, overlaps and differences. Doing Disabil Res. 1997;5:66-87.

3. Rowley E, Wright N, Waring J, Gregoriou K, Chopra A. Protocol for an exploration of knowledge sharing for improved discharge from a mental health ward. BMJ Open. 2014;4:e005176. https://doi.org/10.1136/bmjopen2014-005176.

4. Burns J, Flynn S, Lowe R, Turnbull P, Baird A, Stones P, et al. The National Confidential Inquiry into suicide and homicide by people with mental illness. 2017.

5. Exbrayat S, Coudrot C, Gourdon X, Gay A, Sevos J, Pellet J, et al. Effect of telephone follow-up on repeated suicide attempt in patients discharged from an emergency psychiatry department: a controlled study. BMC Psychiatry. 2017;17. https://doi.org/10.1186/s12888-017-1258-6.

6. Bennewith O, Evans J, Donovan J, Paramasivan S, Owen-Smith A, Hollingworth W, et al. A contact-based intervention for people recently discharged from inpatient psychiatric care: a pilot study. Arch Suicide Res. 2014. https://doi.org/10.1080/13811118.2013.838196.

7. Forchuk C, Reynolds W, Sharkey S, Martin MLJE. The transitional discharge model: comparing implementation in Canada and Scotland. J Psychosoc Nurs Ment Health Serv. 2007;45:31-8.

8. Tomita A, Lukens EP, Herman DB. Mediation analysis of critical time intervention for persons living with serious mental illnesses: assessing the role of family relations in reducing psychiatric rehospitalization. Psychiatr Rehabil J. 2014;37:4-10. https://doi.org/10.1037/prj0000015.

9. Herman D, Conover S, Gorroochurn P, Hinterland K, Hoepner L, Susser ES. A randomized trial of critical time intervention to prevent homelessness in persons with severe mental illness following institutional discharge. Psychiatr Serv. 2011;62:713-9. https://doi.org/10.1176/appi.ps.62.7.713.A.

10. Forchuk C, Godin M, Hoch JS, Kingston-MacClure S, Jeng M, Mo S, Puddy L, et al. Preventing homelessness after discharge from psychiatric wards: 
perspectives of consumers and staff. J Psychosoc Nurs Ment Health Serv. 2013;51:24-31. https://doi.org/10.3928/02793695-20130130-02.

11. Abraham O, Myers MN, Brothers AL, Montgomery J, Norman BA, Fabian T. Assessing need for pharmacist involvement to improve care coordination for patients on LAl antipsychotics transitioning from hospital to home: a work system approach. Res Soc Adm Pharm. 2017;13:1004-13. https://doi. org/10.1016/j.sapharm.2017.02.006.

12. Shaw H, Mackie CA, Sharkie I. Evaluation of effect of pharmacy discharge planning on medication problems experienced by discharged acute admission mental health patients. Int J Pharm Pract. 2000;8:144-53.

13. Attfield J, Brown S, Carter T, Callaghan P. A retrospective case comparison study of the relationship between an integrated care pathway for people diagnosed with schizophrenia in acute mental health care and service users' length of stay, readmission rates and follow-up within 7days of discharge. J Psychiatr Ment Health Nurs. 2017;24:348-57. https://doi.org/10. 1111/jpm.12386.

14. Forchuk C, Martin ML, Jensen E, Ouseley S, Sealy P, Beal G, et al. Integrating an evidence-based intervention into clinical practice: "Transitional relationship model.". J Psychiatr Ment Health Nurs. 2013;20:584-94. https:// doi.org/10.1111/j.1365-2850.2012.01956.x.

15. Vogel I. Review of the use of 'theory of change' in international development. Review Report. 2012. https://doi.org/10.1177/ 109821400302400102.

16. Breuer $E$, Lee L, De Silva M, Lund C. Using theory of change to design and evaluate public health interventions: a systematic review. Implement Sci. 2015;11:63. https://doi.org/10.1186/s13012-016-0422-6.

17. Weiss $\mathrm{CH}$. Nothing as practical as good theory: exploring theory-based evaluation for comprehensive community initiatives for children and families. New Approaches to Eval Community Initiat Concepts, Methods, Context. 1995:65-92. https://doi.org/10.1177/1356389003094007.

18. Vigod SN, Kurdyak PA, Dennis C-L, Leszcz T, Taylor VH, Blumberger DM, et al. Transitional interventions to reduce early psychiatric readmissions in adults: systematic review. Br J Psychiatry. 2013;202:187-94. https://doi.org/ 10.1192/bjp.bp.112.115030.

19. Steffen S, Kösters M, Becker T, Puschner B. Discharge planning in mental health care: a systematic review of the recent literature. Acta Psychiatr Scand. 2009;120:1-9. https://doi.org/10.1111/j.1600-0447.2009.01373.x.

20. Hong QN, Fàbregues S, Bartlett G, Boardman F, Cargo M, Dagenais P, et al. The mixed methods appraisal tool (MMAT) version 2018 for information professionals and researchers. Educ Inf. 2018;34:285-91. https://doi.org/10. 3233/EFI-180221.

21. Popay J, Arai L, Rodgers M, Britten N. Guidance on the conduct of narrative synthesis in systematic reviews: A product from the ESRC Methods Programme. 2006. doi:https://doi.org/10.13140/2.1.1018.4643.

22. Rodgers $M$, Sowden A, Petticrew M, Arai L, Roberts $H$, Britten N, et al. Testing methodological guidance on the conduct of narrative synthesis in systematic reviews: effectiveness of interventions to promote smoke alarm ownership and function. Evaluation. 2009;15:49-73. https://doi.org/10.1177/ 1356389008097871.

23. Benzies KM, Premji S, Hayden KA, Serrett K. State-of-the-evidence reviews: advantages and challenges of including grey literature. Worldviews EvidenceBased Nurs. 2006;3:55-61. https://doi.org/10.1111/j.1741-6787.2006.00051.x.

24. Kasprow WJ, Rosenheck RA. Outcomes of critical time intervention case Management of Homeless Veterans after Psychiatric Hospitalization. Psychiatr Serv. 2014;58:929-35. https://doi.org/10.1176/ps.2007.58.7.929.

25. Tomita A, Herman DB. The impact of critical time intervention in reducing psychiatric rehospitalization after hospital discharge. Psychiatr Serv. 2012;63: 935-7. https://doi.org/10.1176/appi.ps.201100468.

26. Shaffer SL, Hutchison SL, Ayers AM, Goldberg RW, Herman D, Duch DA, et al. Brief critical time intervention to reduce psychiatric rehospitalization. Psychiatr Serv. 2015;66:1155-61. https://doi.org/10.1176/appi.ps.201400362.

27. Chen F. Developing community support for homeless people with mental illness in transition. Community Ment Health J. 2014;50:520-30. https://doi. org/10.1007/s10597-013-9641-3

28. Forchuk C, Martin ML, Chan YL, Jensen E. Therapeutic relationships: from psychiatric hospital to community. J Psychiatr Ment Health Nurs. 2005;12: 556-64. https://doi.org/10.1111/j.1365-2850.2005.00873.x.

29. Reynolds W, Lauder W, Sharkey S, Maciver S, Veitch T, Cameron D. The effects of a transitional discharge model for psychiatric patients. J Psychiatr Ment Health Nurs. 2004;11:82-8. https://doi.org/10.1111/j.13652850.2004.00692.x.
30. Scanlan JN, Hancock N, Honey A. Evaluation of a peer-delivered, transitional and post-discharge support program following psychiatric hospitalisation. BMC Psychiatry. 2017;17. https://doi.org/10.1186/s12888-017-1469-x.

31. Lawn S, Smith A, Hunter K. Mental health peer support for hospital avoidance and early discharge: an Australian example of consumer driven and operated service. J Ment Health. 2008;17:498-508. https://doi.org/10. 1080/09638230701530242.

32. Simpson A, Flood C, Rowe J, Quigley J, Henry S, Hall C, et al. Results of a pilot randomised controlled trial to measure the clinical and cost effectiveness of peer support in increasing hope and quality of life in mental health patients discharged from hospital in the UK. BMC Psychiatry. 2014;14. https://doi.org/10.1186/1471-244X-14-30.

33. Botha UA, Coetzee M, Koen L, Niehaus DJH. An attempt to stem the tide: exploring the effect of a 90-day transitional care intervention on readmissions to an acute male psychiatric unit in South Africa. Arch Psychiatr Nurs. 2018;32:384-9. https://doi.org/10.1016/j.apnu.2017.12.002.

34. De Leo D, Heller T. Intensive case management in suicide attempters following discharge from inpatient psychiatric care. Aust J Prim Health. 2007;13:49-58. https://doi.org/10.1071/PY07038.

35. Bauer S, Okon E, Meermann R, Stephanie K. Technology-enhanced maintenance of treatment gains in eating disorders: Efficacy of an intervention delivered via text messaging. J Consult Clin Psychol. 2012;80: 700-6. https://doi.org/10.1037/a0028030.

36. D'Souza R. Improving treatment adherence and longitudinal outcomes in patients with a serious mental illness by using telemedicine. J Telemed Telecare. 2002;8(Suppl 2):113-5.

37. Walker SA, Naji SA, Howie FL, Andrew J, Cameron IM, Eagles JM. The effectiveness of psychiatric discharge coordinators. Prim Care Psychiatry. 2000;6:149-53. https://doi.org/10.1185/135525700543370.

38. Hengartner MP, Passalacqua S, Heim G, Andreae A, Rössler W, von Wyl A. The post-discharge network coordination programme: a randomized controlled trial to evaluate the efficacy of an intervention aimed at reducing rehospitalizations and improving mental health. Front Psychiatry. 2016;7. https://doi.org/10.3389/fpsyt.2016.00027.

39. Hengartner MP, Klauser M, Heim G, Passalacqua S, Andreae A, Rössler W, et al. Introduction of a psychosocial post-discharge intervention program aimed at reducing psychiatric Rehospitalization rates and at improving mental health and functioning. Perspect Psychiatr Care. 2017;53:10-5. https://doi.org/10.1111/ppc.12131.

40. Jensen E, Chapman P, Davis A, Forchuk C, Seymour B, Witcher P, et al. An evaluation of community-based discharge planning in acute mental health care. Can J Community Ment Heal. 2010;29:111-24. https://doi.org/10.7870/ cjcmh-2010-0038.

41. Hampson M, Johnson H, Croudace TJ. The development of a community link team providing intensive comunity support to facilitate early discharge from acute psychiatric care. J Ment Health. 2000;9:517-26.

42. Bonsack C, Golay P, Manetti SG, Gebel S, Ferrari P, Besse C, et al. Linking primary and secondary care after psychiatric hospitalization: comparison between transitional case management setting and routine care for common mental disorders. Front Psychiatry. 2016;7.

43. Virgolesi M, Pucciarelli G, Colantoni AM, D'Andrea F, Di Donato B, Giorgi $F$, et al. The effectiveness of a nursing discharge programme to improve medication adherence and patient satisfaction in the psychiatric intensive care unit. J Clin Nurs. 2017;26:4456-66. https://doi. org/10.1111/jocn.13776.

44. Li Z, Arthur D. Family education for people with schizophrenia in Beijing, China: Randomised controlled trial. Br J Psychiatry. 2005;187:339-45. https:// doi.org/10.1192/bjp.187.4.339.

45. Khaleghparast S, Ghanbari B, Kahani S, Malakouti K, SeyedAlinaghi S, Sudhinaraset $M$. The effectiveness of discharge planning on the knowledge, clinical symptoms and hospitalisation frequency of persons with schizophrenia: a longitudinal study in two hospitals in Tehran, Iran. J Clin Nurs. 2014;23:2215-22. https://doi.org/10.1111/jocn.12499.

46. Karniel-Lauer E, Szor H, Livne S, Melamed Y, Spiro S, Elizur A. The "re-entry group"' - a transitional therapeutic framework for mentally ill patients discharged from the hospital to community clinics. Can J Psychiatr. 2000;45: 837-9. https://doi.org/10.1177/070674370004500909.

47. Sato S, Ikebuchi E, Anzai N, Inoue S. Effects of psychosocial program for preparing long-term hospitalized patients with schizophrenia for discharge from hospital: randomized controlled trial. Pscyhiatry Clin Neurosci. 2012;66: 474-81. https://doi.org/10.1111/j.1440-1819.2012.02393.x. 
48. Hegedüs A, Kozel B, Fankhauser N, Needham I, Behrens J. Outcomes and feasibility of the short transitional intervention in psychiatry in improving the transition from inpatient treatment to the community: a pilot study. Int J Ment Health Nurs. 2018;27:571-80. https://doi.org/10.1111/inm.12338.

49. Puschner B, Baumgartner I, Loos S, Völker K, Ramacher M, Sohla K, et al. Cost-effectiveness of needs-oriented discharge planning in high utilizers of mental health care. Psychiatr Prax. 2012;39:381-7. https://doi.org/10.1055/s0032-1327188

50. Forchuk C, MacClure SK, Van Beers M, Smith C, Csiernik R, Hoch J, et al. Developing and testing an intervention to prevent homelessness among individuals discharged from psychiatric wards to shelters and "no fixed address.". J Psychiatr Ment Health Nurs. 2008;15:569-75. https://doi.org/10. 1111/j.1365-2850.2008.01266.x.

51. Rose LE, Gerson L, Carbo C. Transitional care for seriously mentally ill persons: A pilot study. Arch Psychiatr Nurs. 2007;21:297-308. https://doi.org/ 10.1016/j.apnu.2007.06.010

52. Hanrahan NP, Solomon P, Hurford MO. A pilot randomized control trial: testing a transitional care model for acute psychiatric conditions. J Am Psychiatr Nurses Assoc. 2014;20:315-27. https://doi.org/10.1177/ 1078390314552190.

53. Attfield J, Brown S, Carter T, Callaghan P. A retrospective case comparison study of the relationship between an integrated care pathway for people diagnosed with schizophrenia in acute mental health care and service users' length of stay, readmission rates and follow-up within 7 days of discharge. J Psychiatr Ment Health Nurs. 2017;24:348-57. https://doi.org/10. 1111/jpm.12386.

54. Juven-Wetzler A, Bar-Ziv D, Cwikel-Hamzany S, Abudy A, Peri N, Zohar J. A pilot study of the "continuation of care" model in "revolving-door" patients. Eur Psychiatry. 2012;27:229-33. https://doi.org/10.1016/j.eurpsy.2011.05.003.

55. Kidd SA, Virdee G, Mihalakakos G, McKinney C, Feingold L, Collins A, et al. The welcome basket revisited: Testing the feasibility of a brief peer support intervention to facilitate transition from hospital to community. Psychiatr Rehabil J. 2016;39:335-42. https://doi.org/10.1037/prj0000235.

56. Smelson D, Kalman D, Losonczy MF, Kline A, Sambamoorthi U, St. Hill L, et al. A brief treatment engagement intervention for individuals with cooccurring mental illness and substance use disorders: Results of a randomized clinical trial. Community Ment Health J. 2012;48:127-32. https:// doi.org/10.1007/s10597-010-9346-9.

57. Ghadiri Vasfi M, Moradi-Lakeh M, Esmaeili N, Soleimani N, Hajebi A. Efficacy of aftercare Services for People with Severe Mental Disorders in Iran: a randomized controlled trial. Psychiatr Serv. 2015;66:373-80. https://doi.org/ 10.1176/appi.ps.201400111.

58. Khanbhai Y, Nance M, Smith D. The development and implementation of a discharge checklist for psychiatric inpatients: a pilot study. Australas Psychiatry. 2018;26:259-62. https://doi.org/10.1177/1039856217751987.

59. Au M, Kehn M, Ireys H, Blyler C, Brown J. Care coordinators in integrated care: burnout risk, perceived supports, and job satisfaction. Am J Prev Med. 2018;54:S250-7. https://doi.org/10.1016/j.amepre.2018.01.044.

60. Joynt KE, Jha AK. Thirty-day readmissions - truth and consequences. $N$ Engl J Med. 2012;366:1366-9. https://doi.org/10.1056/nejmp1201598.

61. Taylor C, Holsinger B, Flanagan J V, Ayers AM, Hutchison SL, Terhorst L. Effectiveness of a brief care management intervention for reducing psychiatric hospitalization readmissions. J Behav Health Serv Res 2016;43: 262-71. doi:https://doi.org/10.1007/s11414-014-9400-4.

62. D'Souza R, Hustig H, Montgomery W. Telehealth in rural and remote mental health: a review of the use of telepsychiatry in education, training, treatment, discharge planning and a health economic evaluation of the telemedicine service. Aust N Z J Psychiatry. 2001;35:A7.

63. Puschner B, Steffen S, Völker KA, Spitzer C, Gaebel W, Janssen B, et al. Needs-oriented discharge planning for high utilisers of psychiatric services: multicentre randomised controlled trial. Epidemiol Psychiatr Sci. 2011;20: 181-92. https://doi.org/10.1017/S2045796011000278.

64. Kisely S, Wyder M, Dietrich J, Robinson G, Siskind D, Crompton D. Motivational aftercare planning to better care: Applying the principles of advanced directives and motivational interviewing to discharge planning for people with mental illness. Int J Ment Health Nurs 2017;26:41-8. doi: https://doi.org/10.1111/inm.12261.

65. NICE. Transition between inpatient hospital settings and community or care home settings for adults with social care needs | Guidance and guidelines | NICE. 2015.
66. National Institute for Health and Care Excellence. Transition between inpatient mental health settings and community or care home settings | Guidance and guidelines | NICE. NICE; 2016.

67. Waring J, Bishop S, Marshall F, Tyler N, Vickers R. An ethnographic study comparing approaches to inter-professional knowledge sharing and learning in discharge planning and care transitions. J Health Organ Manag. 2019.

68. Department of Health. Department of Health. (1990). The Care Programme Approach for people with a mental illness, referred to specialist psychiatric services 1990.

69. Okunogbe A, Meredith LS, Chang ET, Simon A, Stockdale SE, Rubenstein LV. Care coordination and provider stress in primary care management of highrisk patients. J Gen Intern Med. 2018;33:65-71. https://doi.org/10.1007/ s11606-017-4186-8.

70. Lang K. Outcomes for mental health services: what really matters? | HSR UK; 2019.

71. Friebel R, Hauck K, Aylin P, Steventon A. National trends in emergency readmission rates: a longitudinal analysis of administrative data for England between 2006 and 2016. BMJ Open. 2018;8:e020325. https://doi.org/10. 1136/bmjopen-2017-020325.

72. Reed JE, McNicholas C, Woodcock T, Issen L, Bell D. Designing quality improvement initiatives: the action effect method, a structured approach to identifying and articulating programme theory. BMJ Qual Saf. 2014;23:10408. https://doi.org/10.1136/BMJQS-2014-003103.

73. van Beuzekom M, Boer F, Akerboom S, Hudson P. Patient safety: latent risk factors. Br J Anaesth. 2010;105:52-9. https://doi.org/10.1093/bja/aeq135.

74. Reason J. Understanding adverse events: human factors. BMJ Qual Saf. 1995:4:80-9.

75. Waring J, Bishop S, Marshall F. A qualitative study of professional and carer perceptions of the threats to safe hospital discharge for stroke and hip fracture patients in the English national health service. BMC Health Serv Res. 2016;16:297. https://doi.org/10.1186/s12913-016-1568-2.

76. Payton AR. Mental health, mental illness, and psychological distress: same continuum or distinct phenomena? J Health Soc Behav. 2009;50:213-27. https://doi.org/10.1177/002214650905000207.

77. Aitken LM, Chaboyer W, Schuetz M, Joyce C, Macfarlane B. Health status of critically ill trauma patients. J Clin Nurs. 2014;23:704-15. https://doi.org/10. 1111/jocn.12026.

78. A goal-oriented limited-duration approach for borderline personality disorder during brief inpatient hospitalizations. Psychotherapy 2012;49:50218. doi:https://doi.org/10.1037/a0026128.

79. Davidoff F, Dixon-Woods M, Leviton L, Michie S. Demystifying theory and its use in improvement. BMJ Qual Saf. 2015;24:228-38. https://doi.org/10.1136/ bmjas-2014-003627.

80. Harvey C, Brophy L, Tibble H, Killaspy H, Spittal MJ, Hamilton B, et al. Prevention and recovery Care Services in Australia: developing a state-wide typology of a subacute residential mental health service model. Front Psychiatry. 2019;10:383. https://doi.org/10.3389/fpsyt.2019.00383.

\section{Publisher's Note}

Springer Nature remains neutral with regard to jurisdictional claims in published maps and institutional affiliations.

\section{Ready to submit your research? Choose BMC and benefit from:}

- fast, convenient online submission

- thorough peer review by experienced researchers in your field

- rapid publication on acceptance

- support for research data, including large and complex data types

- gold Open Access which fosters wider collaboration and increased citations

- maximum visibility for your research: over $100 \mathrm{M}$ website views per year

At BMC, research is always in progress.

Learn more biomedcentral.com/submission 